\title{
On classical solutions of Rayleigh-Taylor instability in inhomogeneous viscoelastic fluids
}

\author{
Zhidan $\operatorname{Tan}^{1}$ and Weiwei Wang ${ }^{1,2^{*}}$
}

\author{
"Correspondence: \\ wei.wei.84@163.com \\ ${ }^{1}$ College of Mathematics and \\ Computer Science, Fuzhou \\ University, Fuzhou, China \\ ${ }^{2}$ Key Laboratory of Operations \\ Research and Control of Universities \\ in Fujian, Fuzhou, China
}

\begin{abstract}
We study the nonlinear Rayleigh-Taylor (RT) instability of an inhomogeneous incompressible viscoelastic fluid in a bounded domain. It is well known that there exist strong solutions of RT instability in $H^{2}$-norm in inhomogeneous incompressible viscoelastic fluids, when the elasticity coefficient $\boldsymbol{\kappa}$ is less than some threshold $\kappa_{\mathrm{C}}$. In this paper, we prove the existence of classical solutions of RT instability in $L^{1}$-norm in Lagrangian coordinates based on a bootstrap instability method with finer analysis, if $\kappa<\kappa_{\mathrm{C}}$. Moreover, we also get classical solutions of RT instability in $L^{1}$-norm in Eulerian coordinates by further applying an inverse transformation of Lagrangian coordinates.
\end{abstract}

Keywords: Rayleigh-Taylor instability; Inhomogeneous viscoelastic fluids; Bootstrap instability method

\section{Introduction}

Consider two completely plane-parallel layers of immiscible fluids, the heavier on top of the lighter one and both subject to the earth's gravity. In this case, the equilibrium state is unstable to sustain small disturbances, and this unstable disturbance will grow and lead to a release of potential energy, as the heavier fluid moves down under the gravitational force and the lighter one is displaced upwards. This phenomenon was first studied by Rayleigh and then Taylor [37], and is therefore called the Rayleigh-Taylor instability. In 2003, Hwang and Guo [16] mathematically proved the existence of classical solutions of (nonlinear) RT instability in the sense of $L^{2}$-norm for a 2D nonhomogeneous incompressible inviscid fluid. Then Jiang and Jiang further showed the existence of strong solutions of RT instability for 3D nonhomogeneous incompressible viscous fluids in the sense of $L^{2}$ norm [18]. Recently, Jiang and Zhao further proved the existence of classical solutions of RT instability [28]. Similar results of RT instability were established for stratified incompressible viscous fluids, see [26] for instance.

In this article, we are interested in the RT instability of an inhomogeneous incompressible viscoelastic fluid. The RT instability in viscoelastic fluid has been widely studied, see $[15,25,27]$ for examples. Moreover, the RT instability also has been investigated under other physical factors, such as internal surface tension [12, 17, 41], magnetic fields [4, 5, $19-21,23,24,39,40]$, rotation $[3,6,29]$, and so on. We also refer to [2, 8, 14, 30, 33-36] for related progress in other mathematical problems of hydromechanics. 
Recently, Jiang et al. [25] have proved the existence result of strong solutions of RT instability in an inhomogeneous incompressible viscoelastic fluid. To begin with, let us recall the equations of an inhomogeneous incompressible viscoelastic fluid in the presence of a uniform gravitational field in a bounded domain $\Omega \subset \mathbb{R}^{3}$ :

$$
\left\{\begin{array}{l}
\rho_{t}+v \cdot \nabla \rho=0 \\
\rho v_{t}+\rho v \cdot \nabla v+\nabla p=\mu \Delta v+\kappa \operatorname{div}\left(\rho\left(U U^{\mathrm{T}}-I\right)\right)-g \rho e_{3} \\
U_{t}+v \cdot \nabla U-\nabla v U=0 \\
\operatorname{div} v=0
\end{array}\right.
$$

where $\rho:=\rho(x, t)$ is the density, $v:=v(x, t)$ is the velocity, $p:=p(x, t)$ denotes the hydrodynamic pressure, and $U:=U(x, t)$ is the deformation tensor (a $3 \times 3$ matrix valued function). The constants $\mu>0, \kappa>0$, and $g>0$ stand for the coefficient of shear viscosity, the elasticity coefficient, and the gravitational constant, resp. $e_{3}:=(0,0,1)^{\mathrm{T}}$ represents the vertical unit vector, $I$ the identity matrix, $-\rho g e_{3}$ the gravitational force, and the superscript T the transposition. In system (1.1), equation $(1.1)_{1}$ is the continuity equation, $(1.1)_{2}$ is the balance law of momentum, $(1.1)_{3}$ is the deformation equation, and $(1.1)_{4}$ is the incompressible condition.

To mathematically prove the viscoelastic RT instability, we choose a density profile $\bar{\rho}:=$ $\bar{\rho}\left(x_{3}\right)$ satisfying

$$
\begin{aligned}
& \bar{\rho} \in C^{4}(\Omega), \quad \inf _{x \in \Omega} \bar{\rho}>0, \\
& \left.\bar{\rho}^{\prime}\right|_{x_{3}=x_{3}^{0}}>0 \quad \text { for some } x^{0} \in \Omega,
\end{aligned}
$$

where $^{\prime}:=\mathrm{d} / \mathrm{d} x_{3}$ and $x_{3}^{0}$ denotes the third component of $x^{0} \in \Omega$. It should be noted that condition (1.3) ensures that there is some sub-domain of $\Omega$ in which the heavier part of (viscoelastic) fluid is over the lighter one, leading to the classical RT instability. Then $(\rho, v, U)=(\bar{\rho}, 0, I)$ defines an RT equilibrium state to system (1.1), where the equilibrium pressure profile $\bar{p}$ is defined by

$$
\nabla \bar{p}=-\bar{\rho} g e_{3} .
$$

Now, we denote the perturbation around the RT equilibrium state by

$$
\varrho=\rho-\bar{\rho}, \quad \sigma=p-\bar{p}, \quad V=U-I,
$$

then $(\varrho, v, \sigma, V)$ satisfies the following perturbation equations:

$$
\begin{cases}\varrho_{t}+v \cdot \nabla(\varrho+\bar{\rho})=0 & \text { in } \Omega, \\ (\varrho+\bar{\rho}) v_{t}+(\varrho+\bar{\rho}) v \cdot \nabla v+\nabla \sigma & \text { in } \Omega, \\ =\mu \Delta v+\kappa \operatorname{div}\left((\varrho+\bar{\rho})\left(V+V^{\mathrm{T}}+V V^{\mathrm{T}}\right)\right)-g \varrho e_{3} & \text { in } \Omega, \\ V_{t}+v \cdot \nabla V-\nabla v(I+V)=0 & \text { in } \Omega, \\ \operatorname{div} v=0 & \text { in } \Omega .\end{cases}
$$


For system (1.4), we impose the initial-boundary value conditions:

$$
\left.(\varrho, v, V)\right|_{t=0}=\left(\varrho^{0}, v^{0}, V^{0}\right) \text { and }\left.\quad v\right|_{\partial \Omega}=0 .
$$

The initial-boundary value problem (1.4)-(1.5) is called the VRT problem for an inhomogeneous viscoelastic fluid. In [25], Jiang, Jiang, and Wu proved the existence of strong solutions of RT instability for the VRT problem in $\mathrm{H}^{2}$-norm under an instability condition. In this paper, we further establish the existence of unstable classical solutions in the sense of $L^{1}$-norm for the VRT problem based on a bootstrap instability method by further exploiting some new mathematical techniques. Thus our instability result further improves Jiang, Jiang, and Wu's one in [25].

In general, it is hard to directly show the existence of a unique classical solution to (1.4)(1.5) in $L^{2}(\Omega)$-norm. Therefore, we switch our analysis to Lagrangian coordinates. To this end, we assume that there exists an invertible mapping $\zeta^{0}:=\zeta^{0}(y): \Omega \rightarrow \Omega$, such that $\partial \Omega=\zeta^{0}(\partial \Omega)$ and $\operatorname{det} \nabla \zeta^{0}=1$. We define the flow map $\zeta$ to be the solution to

$$
\begin{cases}\partial_{t} \zeta(y, t)=v(\zeta(y, t), t) & \text { in } \Omega, \\ \zeta(y, 0)=\zeta^{0}(y) & \text { in } \Omega .\end{cases}
$$

We denote the Eulerian coordinates by $(x, t)$ with $x=\zeta(y, t)$, whereas $(y, t) \in \Omega \times \mathbb{R}^{+}$stand for the Lagrangian coordinates. In order to switch back and forth from Lagrangian to Eulerian coordinates, we assume that $\zeta(\cdot, t)$ is invertible and $\Omega=\zeta(\Omega, t)$. In other words, the Eulerian domain of the fluid is the image of $\Omega$ under the mapping $\zeta$. In view of the nonslip boundary condition $\left.v\right|_{\partial \Omega}=0$, we have $\partial \Omega=\zeta(\partial \Omega, t)$. Moreover, by the incompressible condition, we have

$$
\operatorname{det} \nabla \zeta=1 \quad \text { in } \Omega
$$

as well as the initial condition $\operatorname{det} \nabla \zeta^{0}=1$, see [31, Proposition 4.1].

In Lagrangian coordinates, the deformation tensor $\tilde{U}(y, t)$ is defined by a Jacobi matrix of $\zeta(y, t)$ :

$$
\tilde{U}(y, t):=\nabla \zeta(y, t), \quad \text { i.e., } \quad \tilde{U}_{i j}:=\partial_{j} \zeta_{i}(y, t)
$$

Here and in what follows, $\partial_{j}$ denotes the partial derivative with respect to the $j$ th component of the spatial variables. When we study this deformation tensor in Eulerian coordinates, we shall denote it by $U(x, t):=\tilde{U}\left(\zeta^{-1}(x, t), t\right)$. Applying the chain rule, it is easy to see that $U(x, t)$ satisfies the transport equation

$$
\partial_{t} U+v \cdot \nabla U=\nabla v U
$$

Let $\eta:=\zeta-y$, and we define the Lagrangian unknowns by

$$
(\tilde{\varrho}, u, \tilde{p})(y, t)=(\rho, v, p)(\zeta(y, t), t) \quad \text { for }(y, t) \in \Omega \times \mathbb{R}^{+} .
$$


Then, in Lagrangian coordinates, the evolution equations for $\tilde{\varrho}, u$, and $\tilde{p}$ read as follows:

$$
\begin{cases}\eta_{t}=u & \text { in } \Omega, \\ \tilde{\varrho}_{t}=0 & \text { in } \Omega, \\ \tilde{\varrho} u_{t}-\mu \Delta_{\mathcal{A}} u+\nabla_{\mathcal{A}} \tilde{p}=\kappa \operatorname{div}_{\mathcal{A}}\left(\tilde{\varrho}\left(\mathbb{D} \eta+\nabla \eta \nabla \eta^{\mathrm{T}}\right)\right)-\tilde{\varrho} g e_{3} & \text { in } \Omega, \\ \operatorname{div}_{\mathcal{A}} u=0 & \text { in } \Omega\end{cases}
$$

with the initial and boundary conditions

$$
\left.(\eta, \tilde{\varrho}, u)\right|_{t=0}=\left(\eta^{0}, \tilde{\varrho}^{0}, u^{0}\right) \text { and }\left.(u, \eta)\right|_{\partial \Omega}=(0,0) \text {, }
$$

where $\mathbb{D} \eta:=\nabla \eta+\nabla \eta^{T}$.

Next, we shall introduce the notations involving $\mathcal{A}$. The matrix $\mathcal{A}:=\left(\mathcal{A}_{i j}\right)_{3 \times 3}$ is defined via $\mathcal{A}^{\mathrm{T}}=(\nabla \zeta)^{-1}:=\left(\partial_{j} \zeta_{i}\right)_{3 \times 3}^{-1}$ :

$$
\nabla_{\mathcal{A}} w:=\left(\nabla_{\mathcal{A}} w_{1}, \nabla_{\mathcal{A}} w_{2}, \nabla_{\mathcal{A}} w_{3}\right)^{\mathrm{T}} \quad \text { and } \quad \nabla_{\mathcal{A}} w_{i}:=\left(\mathcal{A}_{1 k} \partial_{k} w_{i}, \mathcal{A}_{2 k} \partial_{k} w_{i}, \mathcal{A}_{3 k} \partial_{k} w_{i}\right)^{\mathrm{T}}
$$

for a vector function $w:=\left(w_{1}, w_{2}, w_{3}\right)$, and

$$
\operatorname{div}_{\mathcal{A}}\left(f_{1}, f_{2}, f_{3}\right)=\left(\operatorname{div}_{\mathcal{A}} f_{1}, \operatorname{div}_{\mathcal{A}} f_{2}, \operatorname{div}_{\mathcal{A}} f_{3}\right)^{\mathrm{T}} \quad \text { and } \quad \operatorname{div}_{\mathcal{A}} f_{i}:=\mathcal{A}_{l k} \partial_{k} f_{i l}
$$

for a vector function $f_{i}:=\left(f_{i 1}, f_{i 2}, f_{i 3}\right)^{\mathrm{T}}$. It should be noted that we have used the Einstein convention of summation over repeated indices. In addition, we define $\Delta_{\mathcal{A}} X:=\operatorname{div}_{\mathcal{A}} \nabla_{\mathcal{A}} X$ and $\tilde{\mathcal{A}}:=\mathcal{A}-I$.

Further, we introduce some properties of $\mathcal{A}$. In view of the definition of $\mathcal{A}$ and (1.7), we see that

$$
\mathcal{A}=\left(A_{i j}^{*}\right)_{3 \times 3}
$$

where $A_{i j}^{*}$ is the algebraic complement minor of $(i, j)$ th entry $\left(\partial_{j} \zeta_{i}\right)_{3 \times 3}$. Then we have $\partial_{k} A_{i k}^{*}=$ 0 , which implies two important relations:

$$
\operatorname{div}_{\mathcal{A}} u=\operatorname{div}\left(A^{T} u\right)
$$

and

$$
\operatorname{div}_{\mathcal{A}_{t}} u=\operatorname{div}\left(\mathcal{A}_{t}^{T} u\right)
$$

By virtue of $(1.9)_{2}$, we get $\tilde{\varrho}=\rho^{0}\left(\zeta^{0}\right)$. Thus, if

$$
\rho^{0}\left(\zeta^{0}\right)=\bar{\rho}\left(y_{3}\right),
$$

we have

$$
\tilde{\varrho}=\bar{\rho}\left(y_{3}\right) .
$$


Now we define that

$$
q=\tilde{p}-\bar{p}\left(\zeta_{3}\right)
$$

where $\bar{p}\left(\zeta_{3}\right)$ satisfies the equilibrium state in Lagrangian coordinates

$$
\nabla_{\mathcal{A}} \bar{p}\left(\zeta_{3}\right)=-\bar{\rho}\left(\zeta_{3}\right) g e_{3}
$$

Putting the above two relations into (1.9), we have a so-called transformed VRT problem:

$$
\begin{cases}\eta_{t}=u & \text { in } \Omega, \\ \bar{\rho} u_{t}-\mu \Delta_{\mathcal{A}} u+\nabla_{\mathcal{A}} q=\kappa \operatorname{div}_{\mathcal{A}}\left(\bar{\rho}\left(\mathbb{D} \eta+\nabla \eta \nabla \eta^{\mathrm{T}}\right)\right)+g G e_{3} & \text { in } \Omega, \\ \operatorname{div}_{\mathcal{A}} u=0 & \text { in } \Omega, \\ (\eta, u)=0 & \text { on } \partial \Omega, \\ \left.(\eta, u)\right|_{t=0}=\left(\eta^{0}, u^{0}\right) & \text { in } \Omega,\end{cases}
$$

where $G:=\bar{\rho}\left(y_{3}+\eta_{3}(y, t)\right)-\bar{\rho}\left(y_{3}\right)$.

For the derivation of a priori energy estimates of the transformed VRT problem, we shall rewrite (1.16) as the following nonhomogeneous form, in which the terms on the left-hand side of the equations are linear:

$$
\begin{cases}\eta_{t}=u & \text { in } \Omega, \\ \bar{\rho} u_{t}+\operatorname{div} \mathcal{S}(q, u, \eta)-g \bar{\rho}^{\prime} \eta_{3} e_{3}=\mathcal{N}^{1} & \text { in } \Omega, \\ \operatorname{div} u=-\operatorname{div}_{\tilde{\mathcal{A}}} u & \text { in } \Omega, \\ (\eta, u)=0 & \text { on } \partial \Omega, \\ \left.(\eta, u)\right|_{t=0}=\left(\eta^{0}, u^{0}\right) & \text { in } \Omega,\end{cases}
$$

where we have defined that $\mathcal{S}(q, u, \eta):=q I-\mu \nabla u-\kappa \bar{\rho} \mathbb{D} \eta$ and

$$
\begin{aligned}
\mathcal{N}^{1}:= & \mu\left(\operatorname{div}_{\tilde{\mathcal{A}}} \nabla_{\mathcal{A}} u+\operatorname{div} \nabla_{\tilde{\mathcal{A}}} u\right)-\nabla_{\tilde{\mathcal{A}}} q+\kappa\left(\operatorname{div}_{\tilde{\mathcal{A}}}(\bar{\rho} \mathbb{D} \eta)+\operatorname{div}_{\mathcal{A}}\left(\bar{\rho} \nabla \eta \nabla \eta^{\mathrm{T}}\right)\right) \\
& +g e_{3} \int_{0}^{\eta_{3}(y, t)} \int_{0}^{\tau} \bar{\rho}^{\prime \prime}\left(y_{3}+s\right) \mathrm{d} s \mathrm{~d} \tau,
\end{aligned}
$$

and $\operatorname{div}_{\tilde{\mathcal{A}}}$ and $\Delta_{\tilde{\mathcal{A}}}$ are defined by the definition of $\operatorname{div}_{\mathcal{A}}$ and $\Delta_{\mathcal{A}}$ with $\tilde{\mathcal{A}}$ in place of $\mathcal{A}$, respectively.

By omitting the nonlinear terms $\mathcal{N}^{1}$ and $-\operatorname{div}_{\tilde{\mathcal{A}}} u$ in (1.17), we get a linearized VRT problem as follows:

$$
\begin{cases}\eta_{t}=u & \text { in } \Omega, \\ \bar{\rho} u_{t}+\operatorname{div} \mathcal{S}(q, u, \eta)=g \bar{\rho}^{\prime} \eta_{3} e_{3} & \text { in } \Omega, \\ \operatorname{div} u=0 & \text { in } \Omega, \\ (\eta, u)=0 & \text { on } \partial \Omega, \\ \left.(\eta, u)\right|_{t=0}=\left(\eta^{0}, u^{0}\right) & \text { in } \Omega .\end{cases}
$$


The linearized problem is convenient to analyze in order to have an insight into the physical and mathematical mechanisms of the VRT problem. In fact, using a standard energy method, Jiang et al. [25] found stability and instability criteria of the above linearized VRT problem. Moreover, Jiang et al. [25] further proved that there exists a unique unstable strong solution in the sense of $H^{2}(\Omega)$-norm to the VRT problem, when

$$
\kappa<\kappa_{\mathrm{C}}:=\sup _{w \in H_{\sigma}^{1}} \frac{2 g \int \bar{\rho}^{\prime} w_{3}^{2} \mathrm{~d} x}{\|\sqrt{\bar{\rho}} \mathbb{D} w\|_{L^{2}(\Omega)}^{2}}, \quad H_{\sigma}^{1}:=\left\{w \in H_{0}^{1} \mid \operatorname{div} w=0 \text { in } \Omega\right\} .
$$

Before stating our main result, we shall introduce the following simplified notations:

$$
\begin{aligned}
& \int:=\int_{\Omega}, \quad L^{p}:=L^{p}(\Omega)=W^{0, p}(\Omega), \quad H_{0}^{1}:=W_{0}^{1,2}(\Omega), \quad H^{k}:=W^{k, 2}(\Omega), \\
& H_{\sigma}^{k}:=H_{\sigma}^{1} \cap H^{k}, \quad H_{0}^{k}:=H_{0}^{1} \cap H^{k}, \quad \underline{H}^{k}:=\left\{w \in H^{k} \mid(w)_{\Omega}=0\right\} \\
& \|\cdot\|_{k}:=\|\cdot\|_{H^{k}}, \quad\|(u, q)\|_{\mathrm{S}, k}:=\sqrt{\|u\|_{k+2}^{2}+\|q\|_{k+1}^{2}}, \\
& E(w):=\frac{1}{2}\|\sqrt{\kappa \bar{\rho}} \mathbb{D} w\|_{0}^{2}-g \int \bar{\rho}^{\prime}\left|w_{3}\right|^{2} \mathrm{~d} y, \\
& \mathcal{E}(t):=\|\eta(t)\|_{4}^{2}+\|(u, q)(t)\|_{\mathrm{S}, 1}^{2}+\left\|u_{t}\right\|_{1}^{2}, \\
& \mathcal{D}(t):=\|\eta(t)\|_{4}^{2}+\|(u, q)(t)\|_{\mathrm{S}, 2}^{2}+\left\|\partial_{t}(u, q)\right\|_{\mathrm{S}, 0}^{2}+\left\|u_{t t}\right\|_{0}^{2}, \\
& a \lesssim b \text { means that } a \leq c b \text { for some constant } c
\end{aligned}
$$

where $1 \leq p \leq \infty, k$ is a nonnegative integer, and the positive constant $c$ may depend on the domain occupied by the fluids and other known physical parameters such as $\rho, \mu, g$, and $\kappa$, and vary from line to line.

Next we state our main result in the transformed VRT problem.

Theorem 1.1 Under assumptions (1.2), (1.3), and (1.19), the zero solution to the transformed VRT problem (1.16) is unstable in the Hadamard sense, that is, there are positive constants $\Lambda, m_{0}, \epsilon$, and $\delta_{0}$, and $\left(\tilde{\eta}^{0}, \tilde{u}^{0}, \eta^{\mathrm{r}}, u^{\mathrm{r}}\right) \in H_{\sigma}^{4} \times H_{\sigma}^{3} \times H_{0}^{4} \times H_{0}^{3}$, such that, for any $\delta \in\left(0, \delta_{0}\right)$ and the initial data

$$
\left(\eta^{0}, u^{0}\right):=\delta\left(\tilde{\eta}^{0}, \tilde{u}^{0}\right)+\delta^{2}\left(\eta^{\mathrm{r}}, u^{\mathrm{r}}\right) \in H_{0}^{4} \times H_{0}^{3},
$$

there is a unique classical solution $(\eta, u, q) \in C^{0}\left([0, T), H_{0}^{4} \times H_{0}^{3} \times \underline{H}^{2}\right)$ to the transformed $V R T$ problem satisfying

$$
\left\|\eta_{3}\left(T^{\delta}\right)\right\|_{L^{1}},\left\|\eta_{\mathrm{h}}\left(T^{\delta}\right)\right\|_{L^{1}},\left\|u_{3}\left(T^{\delta}\right)\right\|_{L^{1}},\left\|u_{\mathrm{h}}\left(T^{\delta}\right)\right\|_{L^{1}} \geq \epsilon
$$

for some escape time $T^{\delta}:=\frac{1}{\Lambda} \ln \frac{2 \epsilon}{m_{0} \delta} \in(0, T)$, where $T$ denotes an existence time of the solution $(\eta, u)$. Moreover, the initial data $\left(\eta^{0}, u^{0}\right) \in H_{0}^{4} \times H_{0}^{3}$ satisfies the keeping volume condition $" \operatorname{det}\left(\nabla \eta^{0}+I\right)=1$ in $\Omega$ " and necessary compatibility conditions:

$$
\begin{aligned}
& \operatorname{div}_{\mathcal{A}^{0}} u^{0}=0 \text { in } \Omega, \\
& \operatorname{div}_{\mathcal{A}^{0}}\left(u^{0} \cdot \nabla_{\mathcal{A}^{0}} u^{0}+\left(\nabla_{\mathcal{A}^{0}} q^{0}-\mu \Delta_{\mathcal{A}^{0}} u^{0}\right.\right.
\end{aligned}
$$




$$
\begin{aligned}
& \left.\left.-\kappa \operatorname{div}_{\mathcal{A}^{0}}\left(\bar{\rho}\left(\mathbb{D} \eta^{0}+\nabla \eta^{0}\left(\nabla \eta^{0}\right)^{T}\right)\right)-g G^{0} e_{3}\right) / \bar{\rho}\right)=0 \quad \text { in } \Omega, \\
& \nabla_{\mathcal{A}^{0} q^{0}-\mu \Delta_{\mathcal{A}^{0}} u^{0}} \\
& -\kappa \operatorname{div}_{\mathcal{A}^{0}}\left(\bar{\rho}\left(\mathbb{D} \eta^{0}+\nabla \eta^{0}\left(\nabla \eta^{0}\right)^{T}\right)\right)-g G^{0} e_{3}=0 \quad \text { on } \partial \Omega,
\end{aligned}
$$

where $G^{0}=\bar{\rho}\left(y_{3}+\eta_{0}^{3}\right)-\bar{\rho}\left(y_{3}\right)$, and $\mathcal{A}^{0}$ denotes the initial data of $\mathcal{A}$ and is defined by $\eta^{0}$ (it should be noted that we automatically have $\operatorname{det}(\nabla \eta+I)=1$ due to the keeping volume condition).

Remark 1.1 It should be noted that the solution $(\eta, u, q)$ in the above theorem enjoys the additional regularity:

$$
\begin{aligned}
& \left(u_{t}, q\right) \in C^{0}\left([0, T), H^{1} \times H^{2}\right), \\
& \left(u, u_{t}, q\right) \in L^{2}\left((0, T), H^{4} \times H^{2} \times H^{3}\right) .
\end{aligned}
$$

Remark 1.2 Compatibility condition (1.20) is deduced from the incompressible condition " $\operatorname{div}_{\mathcal{A}} u=0$ in $\Omega$ ", compatibility condition (1.22) from the compatibility condition " $\left.u_{t}\right|_{t=0}=0$ on $\partial \Omega$ ", and compatibility condition (1.21) from the compatibility condition " $\left.\partial_{t} \operatorname{div}_{\mathcal{A}} u\right|_{t=0}=0$ in $\Omega$ " and the following fact:

$$
\operatorname{div}_{\mathcal{A}_{t}} u=\operatorname{div}\left(\mathcal{A}_{t}^{\mathrm{T}} u\right)=-\operatorname{div}\left(\mathcal{A}^{\mathrm{T}} \nabla \zeta_{t} \mathcal{A}^{\mathrm{T}} u\right)=-\operatorname{div}_{\mathcal{A}}\left(u \cdot \nabla_{\mathcal{A}} u\right)
$$

The above relation can be deduced by using (1.12) and (1.16) .

Remark 1.3 Let $(\eta, u, q)$ be constructed in Theorem 1.1. If $\delta$ is sufficiently small, then, for each fixed $t$,

$$
\begin{aligned}
& \zeta(y, t): \bar{\Omega} \rightarrow \bar{\Omega} \text { is a homeomorphism mapping, } \\
& \zeta(y, t): \Omega \rightarrow \Omega \text { is a } C^{1} \text {-diffeomorphic mapping. }
\end{aligned}
$$

We define

$$
\left\{\begin{array}{l}
\varrho:=\bar{\rho}\left(\zeta_{3}^{-1}(x, t)\right)-\bar{\rho}, \\
v:=u\left(\zeta^{-1}(x, t), t\right), \\
\sigma:=q\left(\zeta^{-1}(x, t), t\right)-\bar{p}, \\
V:=\nabla \zeta\left(\zeta^{-1}(x, t), t\right)-I,
\end{array}\right.
$$

where $\zeta_{3}^{-1}$ denotes the third component of $\zeta^{-1}$. Then it is easy to verify that $(\varrho, v, V)$ is a unique unstable solution to VRT problem (1.4)-(1.5); meanwhile, we can see that the RT instability can occur in the VRT problem, when $\kappa<\kappa_{\mathrm{C}}$.

The proof of Theorem 1.1 is based on a bootstrap method, which has its origin in Guo and Strauss's articles and has been developed by Friedlander [10,11] and other authors. At present, many versions of bootstrap methods have been established in the study of dynamical instability of various physical models, see [7,9-11] for examples. In this article, 
we adopt the version of a bootstrap methods in [9, Lemma 1.1] and [28], thus the proof of Theorem 1.1 can be divided into five steps.

Firstly, in Sect. 2, we construct linear unstable solutions to the linearized VRT problem; this can be achieved by the modified variational method as in [12, 18, 38, 42]. Secondly, in Sect. 3, we shall establish a Gronwall-type energy inequality for the transformed VRT problem by using the energy method in [20]. Thirdly, in Sect. 4, we want to use the initial data for the linearized problem to construct the initial data for the corresponding nonlinear problem as in [9, Lemma 1.1], but unfortunately the initial data of the linearized and the transformed VRT problems have to satisfy different compatibility conditions. To overcome this difficulty, we use the existence theory of Stokes problem and the iterative technique to modify the initial data of solutions for the linearized VRT problem. Thus the obtained modified initial data satisfy compatibility conditions (1.20)-(1.22) and keeping volume condition and are close to the initial data of the linearized VRT problem. Fourthly, in Sect. 5, we deduce the error estimates between the solutions of the linearized and the transformed VRT problems based on a method of largest growth rate as in [16]. We shall use the existence theory of Stokes problem again to modify the error function $u^{d}$ (i.e., the error between the nonlinear and linear solutions concerning $u$ ), which does not enjoy the divergence-free condition (i.e., $\operatorname{div} u^{d}=0$ ). Then the method of largest rate can be used to deduce the desired error estimates. Finally, we show the existence of an escape time and thus obtain Theorem 1.1.

\section{Linear instability}

The modified variational method of ordinary differential equations (ODEs) was firstly found in [12], and later it was extended to partial differential equations (PDEs). Exploiting the modified variational method of PDEs (see $[18,19]$ ), we can construct unstable solutions to the linearized VRT problem.

Proposition 2.1 Under assumption (1.19), there is an unstable solution

$$
(\eta, u, q):=e^{\Lambda t}(w / \Lambda, w, \beta)
$$

to the linearized VRT problem (1.18), where $(w, \beta) \in\left(\mathcal{A} \cap H^{\infty}\right) \times\left(\underline{H}^{1} \cap H^{\infty}\right)$ solves the boundary value problem

$$
\begin{cases}\Lambda^{2} \bar{\rho} w=-\operatorname{div} \mathcal{S}(\Lambda \beta, \Lambda w, w)+g \bar{\rho}^{\prime} w_{3} e_{3} & \text { in } \Omega, \\ \operatorname{div} w=0 & \text { in } \Omega, \\ w=0 & \text { on } \partial \Omega\end{cases}
$$

with a largest growth rate $\Lambda>0$ satisfying

$$
\Lambda^{2}=-\inf _{\varpi \in \mathcal{A}}\left(E(\varpi)+\Lambda \mu\|\nabla \varpi\|_{0}^{2}\right)
$$

where $\mathcal{A}:=\left\{\varpi \in H_{\sigma}^{1} \mid\|\sqrt{\bar{\rho}} \varpi\|_{0}^{2}=1\right\}$. Moreover,

$$
w_{3} \neq 0, \quad w_{\mathrm{h}} \neq 0 \quad \text { in } \Omega \text {. }
$$


Proof We show the proof of Proposition 2.1 by three steps.

(1) At the beginning, we show the existence of classical solutions to the modified problem

$$
\begin{cases}\operatorname{div} \mathcal{S}(s \beta, s w, w)-g \bar{\rho}^{\prime} w_{3} e_{3}=\alpha(s) \bar{\rho} w & \text { in } \Omega, \\ \operatorname{div} w=0 & \text { in } \Omega, \\ w=0 & \text { on } \partial \Omega\end{cases}
$$

where $s>0$ is any given.

To this end, we consider the variational problem of the energy functional

$$
\alpha(s):=\inf _{\varpi \in \mathcal{A}}(E(\varpi)+s F(\varpi))
$$

for given $s>0$, where we have defined that $F(\varpi):=\mu\|\nabla \varpi\|_{0}^{2}$. By the definition of $E(\varpi)$ and $\varpi \in \mathcal{A}$, we can see that $E(\varpi)+s F(\varpi)$ has a minimizing sequence $\left\{w^{n}\right\}_{n=1}^{\infty} \subset \mathcal{A}$, which satisfies $\left\|\sqrt{\bar{\rho}} w^{n}\right\|_{0}=1$ and $\left\|w^{n}\right\|_{1} \leq c$ with $c$ independent of $n$. Moreover, we have

$$
\alpha(s)=\lim _{n \rightarrow \infty}\left(E\left(\varpi^{n}\right)+s F\left(\varpi^{n}\right)\right) .
$$

By the well-known Rellich-Kondrachov compactness theorem, there exist a subsequence(still labeled by $w^{n}$ ) and a function $w \in \mathcal{A}$, such that $w^{n} \rightarrow w$ in $H_{\sigma}^{1}, w^{n} \rightarrow w$ in $L^{2}$. Hence, by the lower semi-continuity of weak convergence, one has

$$
\alpha(s)=\liminf _{n \rightarrow \infty}\left(E\left(w^{n}\right)+s F\left(w^{n}\right)\right) \geq E(w)+s F(w) \geq \alpha(s) .
$$

It is easy to see that $w$ constructed above is the minimum point of the energy functional $(E(\varpi)+s F(\varpi)) /\|\sqrt{\bar{\rho}} \varpi\|_{0}^{2}$ with respect to $\varpi \in H_{\sigma}^{1}$, and

$$
\alpha(s)=(E(w)+s F(w)) /\|\sqrt{\bar{\rho}} w\|_{0}^{2} .
$$

Thus, we can get that the point $t=0$ is the minimum point of the function

$$
I(t):=E(w+t \varphi)+s F(w+t \varpi)-\alpha(s) \int \alpha \bar{\rho}|w+t w|^{2} \mathrm{~d} y \in C^{1}(\mathbb{R})
$$

for any given $\varphi \in H_{\sigma}^{1}$, and $I^{\prime}(0)=0$. So, we directly have the weak form:

$$
g \int \bar{\rho}^{\prime} w_{3} \varphi_{3} \mathrm{~d} y-\int(s \mu+\kappa \bar{\rho}) \mathbb{D} w: \nabla \varphi \mathrm{d} y=\alpha(s) \int \bar{\rho} w \cdot \varphi \mathrm{d} y .
$$

From (2.6) we can see that $w$ is the weak solution of modified problem (2.4).

To improve the regularity of the weak solution $w$, we rewrite (2.4) as the following Stokes problem:

$$
\begin{cases}s \nabla \beta-\Delta \varphi=-\alpha(s) \bar{\rho} w+g \bar{\rho}^{\prime} w_{3} e_{3}+\kappa\left(\bar{\rho}^{\prime} \nabla w_{3}-\bar{\rho}^{\prime} \partial_{3} w-\bar{\rho}^{\prime \prime} w\right) & \text { in } \Omega, \\ \operatorname{div} \varphi=\kappa \bar{\rho}^{\prime} w_{3} & \text { in } \Omega, \\ \varphi=0 & \text { on } \partial \Omega\end{cases}
$$


where $\varphi:=(s \mu+\kappa \bar{\rho}) w$. Applying the existence theory of Stokes problem, we get $w$ is a solution to boundary value problem (2.4) with an associated pressure $\beta$, and $(w, \beta) \in H_{0}^{2} \times$ $H^{1}$. Thus, using the existence theory of Stokes problem again, and a standard bootstrap method, we can further deduce that $(w, \beta) \in\left(\mathcal{A} \cap H^{\infty}\right) \times H^{\infty}$.

(2) We assert that

$$
\begin{aligned}
& \alpha\left(s_{2}\right)>\alpha\left(s_{1}\right) \quad \text { for any } s_{2}>s_{1}>0, \\
& \alpha(s) \in C_{\mathrm{loc}}^{0,1}(0, \infty), \\
& \alpha(s)<0 \text { on some interval }\left(0, c_{2}\right), \\
& \alpha(s)>0 \text { on some interval }\left(c_{3}, \infty\right) .
\end{aligned}
$$

To begin with, we verify (2.7). For given $s_{2}>s_{1}$, then there exists $v^{s_{2}} \in \mathcal{A}$ such that

$$
\alpha\left(s_{2}\right)=E\left(v^{s_{2}}\right)+s_{2} F\left(v^{s_{2}}\right) .
$$

Thus,

$$
\alpha\left(s_{1}\right) \leq E\left(v^{s_{2}}\right)+s_{1} F\left(v^{s_{2}}\right)=\alpha\left(s_{2}\right)+\left(s_{1}-s_{2}\right) F\left(v^{s_{2}}\right)<\alpha\left(s_{2}\right),
$$

which yields (2.7).

Next we turn to proving (2.8). Choose a bounded interval $[a, b] \subset(0, \infty)$, then, for any $s \in$ $[a, b]$, there exists a function $v^{s}$ satisfying $\alpha(s)=E\left(v^{s}\right)+s F\left(v^{s}\right)$. Thus, by the monotonicity (2.7), we have

$$
\alpha(b)-a F\left(v^{s}\right) / 2 \geq E\left(v^{s}\right)+s F\left(v^{s}\right) / 2 \geq \alpha(s / 2) \geq \alpha(a / 2),
$$

which yields

$$
F\left(v^{s}\right) \leq 2(\alpha(b)-\alpha(a / 2)) / a=: K \quad \text { for any } s \in[a, b] .
$$

Thus, for any $s_{1}, s_{2} \in[a, b]$,

$$
\alpha\left(s_{1}\right)-\alpha\left(s_{2}\right) \leq E\left(v^{s_{2}}\right)+s_{1} F\left(v^{s_{2}}\right)-\left(E\left(v^{s_{2}}\right)+s_{2} F\left(v^{s_{2}}\right)\right) \leq K\left|s_{2}-s_{1}\right|
$$

and

$$
\alpha\left(s_{2}\right)-\alpha\left(s_{1}\right) \leq K\left|s_{2}-s_{1}\right|,
$$

which directly imply $\left|\alpha\left(s_{1}\right)-\alpha\left(s_{2}\right)\right| \leq K\left|s_{2}-s_{1}\right|$. Hence (2.8) holds.

Finally, (2.9) and (2.10) can be deduced from the definition of $\alpha$ and assumption (1.19) by Friedrichs's inequality.

(3) We turn to constructing an interval for fixed point: Let

$$
\mathfrak{I}:=\sup \{\text { all the real constant } s \text {, which satisfy that } \alpha(\tau)<0 \text { for any } \tau \in(0, s)\} \text {. }
$$


By virtue of (2.9) and (2.10), it is easy to see that $0<\mathfrak{I}<\infty$. Further, $\alpha(s)<0$ for any $s \in$ $(0, \mathfrak{I})$, by the continuity of $\alpha(s)$, we get

$$
\alpha(\mathfrak{I})=0 .
$$

So, by the monotonicity and the upper boundedness of $\alpha(s)$, we see that

$$
\lim _{s \rightarrow 0} \alpha(s)=\varsigma \text { for some negative constant } \varsigma .
$$

Using (2.11), (2.12), and the continuity of $\alpha(s)$ on $(0, \mathfrak{I})$, we find by a fixed-point argument on $(0, \mathfrak{I})$ that there is unique $\Lambda \in(0, \mathfrak{I})$ satisfying

$$
\Lambda=\sqrt{-\alpha(\Lambda)}=\sqrt{-\inf _{\varpi \in \mathcal{A}}(E(\varpi)+\Lambda F(\varpi))} \in(0, \mathfrak{I}) .
$$

Thus, there is a classical solution $w \in \mathcal{A} \cap H^{\infty}$ to boundary value problem (2.1) with $\Lambda$ constructed above and with $\beta \in \underline{H}^{1} \cap H^{\infty}$. Moreover,

$$
\Lambda=\sqrt{-(E(w)+\Lambda F(w))}>0 .
$$

In addition, (2.3) immediately follows (2.13) and the fact $w \in H_{\sigma}^{2}$. This completes the proof of Proposition 2.1.

\section{Gronwall-type energy inequality of nonlinear solutions}

We derive that any solution of the transformed VRT problem enjoys a Gronwall-type energy inequality by applying a priori estimate method. Let $(\eta, u)$ be a solution of the VRT problem such that

$$
\sup _{0 \leq t<T} \sqrt{\|\eta(t)\|_{4}^{2}+\|u(t)\|_{3}^{2}} \leq \delta \in(0,1)
$$

where $T$ is the existence time of the solution $(\eta, u)$. The smallness of $\delta$ depends on the domain and known parameters in the transformed VRT problem, and it makes sure the solution enjoys fine regularity.

\subsection{Preliminaries}

To begin with, we list some important mathematical results, which will be repeatedly used throughout this paper.

(1) Embedding inequality (see [1, Theorem 4.128]): Let $\Omega \subset \mathbb{R}^{3}$ be a bounded Lipschitz domain, then

$$
\begin{aligned}
& \|f\|_{L^{p}} \lesssim\|f\|_{1} \quad \text { for } 2 \leq p \leq 6, \\
& \|f\|_{C^{0}(\bar{\Omega})}=\|f\|_{L^{\infty}} \lesssim\|f\|_{2} .
\end{aligned}
$$

(2) Interpolation inequality in $H^{j}$ (see [1, 5.2 Theorem]): Let $\Omega$ be a domain in $\mathbb{R}^{3}$ satisfying the cone condition, then, for any $0 \leq j<i, \varepsilon>0$,

$$
\|f\|_{j} \lesssim\|f\|_{0}^{1-j}{ }^{j}\|f\|_{i}^{j} \leq C(\varepsilon)\|f\|_{0}+\varepsilon\|f\|_{i},
$$

where the constant $C(\varepsilon)$ depends on the domain and $\varepsilon$. 
(3) Friedrichs's inequality (see [32, Lemma 1.42]): Let $1 \leq p<\infty$ and $\Omega$ be a bounded Lipschitz domain. Let a set $\Gamma \subset \partial \Omega$ be measurable with respect to the $(N-1)$-dimensional measure $\tilde{\mu}:=\operatorname{meas}_{N-1}$ defined on $\partial \Omega$, and let $\operatorname{meas}_{N-1}(\Gamma)>0$. Then

$$
\|w\|_{W^{1, p}} \lesssim\|\nabla w\|_{L^{p}}^{2}
$$

for all $u \in W^{1, p}(\Omega)$ satisfying that the trace of $u$ on $\Gamma$ is equal to 0 a.e. with respect to the $(N-1)$-dimensional measure $\tilde{\mu}$.

(4) Product estimates (see Sect. 4.1 in [22]): Let $\Omega \in \mathbb{R}^{3}$ be a bounded Lipschitz domain. The functions $f, g$ are defined in $\Omega$. Then

$$
\|f g\|_{i} \lesssim \begin{cases}\|f\|_{1}\|g\|_{1} & \text { for } i=0 ; \\ \|f\|_{i}\|g\|_{2} & \text { for } 0 \leq i \leq 2 \\ \|f\|_{2}\|g\|_{i}+\|f\|_{i}\|g\|_{2} & \text { for } 3 \leq i \leq 5\end{cases}
$$

(5) Korn's inequality (see [13, Lemma 10.7]): Let $\Omega$ be a bounded domain, then, for any $w \in H_{0}^{1}$,

$$
\|w\|_{1}^{2} \lesssim\|\mathbb{D} w\|_{0}^{2}
$$

(6) Existence theory of (steady) Stokes problem (see [41, Lemma A.8]): Denote $\Omega \subset \mathbb{R}^{3}$ to be a bounded domain of $C^{k+2}$-class. Let $\mu$ be constant, $k \geq 0, f^{1} \in H^{k}, f^{2} \in H^{k+1}$, and $f^{3} \in H^{k+3 / 2}(\partial \Omega)$ be given such that

$$
\int_{\Omega} f^{2} \mathrm{~d} x=\int_{\partial \Omega} f^{3} \vec{n} \mathrm{~d} \mathrm{~d} S
$$

where $\vec{n}$ denotes the unit outer normal vector of $\partial \Omega$, then there exists a unique strong solution $(u, q) \in H^{k+2} \times \underline{H}^{k+1}$, which solves

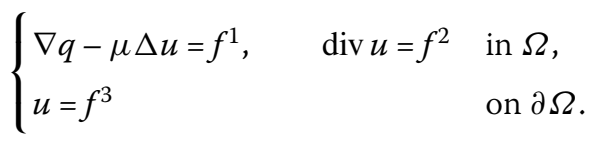

Moreover, any solution $(u, q) \in H^{k+2} \times H^{k+1}$ of (3.6) enjoys the following estimate:

$$
\|u\|_{k+2}+\|q\|_{k+1} \lesssim\left\|f^{1}\right\|_{k}+\left\|f^{2}\right\|_{k+1}+\left\|f^{3}\right\|_{H^{k+3 / 2}(\partial \Omega)} .
$$

Lemma 3.1 Under assumption (3.1), we have:

(1) The estimate for $\mathcal{A}$ : for $0 \leq i \leq 3$ and $0 \leq j \leq 1$,

$$
\begin{aligned}
& \|\mathcal{A}\|_{C^{0}(\bar{\Omega})}+\|\mathcal{A}\|_{3} \lesssim 1, \\
& \left\|\mathcal{A}_{t}\right\|_{i} \lesssim\|u\|_{i+1}, \\
& \left\|\mathcal{A}_{t t}\right\|_{j} \lesssim\left\|\left(u, u_{t}\right)\right\|_{j+1}, \\
& \|\tilde{\mathcal{A}}\|_{i} \lesssim\|\eta\|_{i+1} .
\end{aligned}
$$


(2) The estimate of $\operatorname{div} \eta$ : for $0 \leq i \leq 3$ and $0 \leq j \leq 1$,

$$
\begin{aligned}
&\|\operatorname{div} \eta\|_{i} \lesssim\|\eta\|_{\alpha(i)}\|\eta\|_{i+1}, \\
&\|\operatorname{div} u\|_{i} \lesssim\|\eta\|_{\alpha(i)}\|u\|_{i+1}, \\
&\left\|\operatorname{div} u_{t}\right\|_{j} \lesssim\|\eta\|_{3}\left\|u_{t}\right\|_{j+1}+\|u\|_{3}\|u\|_{j+1},
\end{aligned}
$$

where $\alpha(i)=i+1$ for $2 \leq i, \alpha(i)=3$ for $0 \leq i \leq 1$.

(3) The equivalent estimate

$$
\|w\|_{1} \lesssim\left\|\nabla_{\mathcal{A}} w\right\|_{0} \lesssim\|w\|_{1} \quad \text { for any } w \in H_{0}^{1}
$$

Proof (1) We can derive from $(1.16)_{1}$ and $\operatorname{det}\left(\nabla \eta_{0}+I\right)=1$ that

$$
\operatorname{det}(\nabla \eta+I)=1
$$

Thus, by (1.10), (1.16) ${ }_{1}$, embedding inequality (3.3), and product estimate (3.5), we can easily get (3.8)-(3.10).

By the definition of $\mathcal{A}$ and the smallness of $\delta$, we can derive that

$$
\tilde{\mathcal{A}}^{T}=(\nabla \eta)^{2} \tilde{\mathcal{A}}^{T}+(\nabla \eta)^{2}-\nabla \eta
$$

Consequently, using product estimate (3.5), we get (3.11).

(2) Since $\operatorname{det}(\nabla \eta+I)=1$, and by determinant expansion theorem, we see that

$$
\operatorname{div} \eta=\left(\left(\operatorname{tr}(\nabla \eta)^{2}-\operatorname{div} \eta\right)^{2}\right) / 2-\operatorname{det} \nabla \eta
$$

By (3.1) and product estimate (3.5), we directly deduce (3.12) from the above relation. By (3.9), (3.11), and product estimate (3.5), we can also derive (3.13) and (3.14) from (1.17) 3 .

(3) Exploiting (3.11) and Friedrichs's inequality, we can easily get (3.15).

Lemma 3.2 Estimates of nonlinear terms: Under assumption (3.1):

(1) We have

$$
\begin{aligned}
\left\|\mathcal{N}^{1}\right\|_{i} & \lesssim\|\eta\|_{3}\left(\|(\eta, u)\|_{i+2}+\|q\|_{i+1}\right) \quad \text { for } 0 \leq i \leq 1, \\
\left\|\mathcal{N}^{1}\right\|_{2} & \lesssim\|\eta\|_{4}\left(\|(\eta, u)\|_{4}+\|q\|_{3}\right), \\
\left\|\mathcal{N}_{t}^{1}\right\|_{0} & \lesssim\|(\eta, u)\|_{3}\left(\left\|\left(\eta, u, u_{t}\right)\right\|_{2}+\left\|\left(q, q_{t}\right)\right\|_{1}\right) .
\end{aligned}
$$

(2) In addition,

$$
\left\{\begin{aligned}
\mathcal{N}^{2}:= & \mu\left(\operatorname{div}_{\mathcal{A}} \nabla_{\mathcal{A}_{t}} u+\operatorname{div}_{\mathcal{A}_{t}} \nabla_{\mathcal{A}} u\right)-\nabla_{\mathcal{A}_{t}} q+\kappa \partial_{t}\left(\operatorname{div}_{\mathcal{A}}\left(\bar{\rho} \nabla \eta \nabla \eta^{\mathrm{T}}\right)+\operatorname{div}_{\tilde{\mathcal{A}}}(\bar{\rho} \mathbb{D} \eta)\right) \\
& +g e_{3} u_{3} \int_{0}^{\eta_{3}(y, t)} \bar{\rho}^{\prime \prime}\left(y_{3}+s\right) \mathrm{d} s, \\
\mathcal{N}^{3}:= & \kappa \bar{\rho} \operatorname{div} \eta+\mu \operatorname{div} u
\end{aligned}\right.
$$

we have

$$
\left\|\mathcal{N}^{2}\right\|_{0} \lesssim\|\eta\|_{3}\|(\eta, u)\|_{3}+\|u\|_{2}\left(\|u\|_{3}+\|q\|_{2}\right),
$$




$$
\left\|\mathcal{N}^{3}\right\|_{i+1} \lesssim\|\eta\|_{4}\|(\eta, u)\|_{i+2} \quad \text { for } 0 \leq i \leq 2
$$

Proof Using (1.17) 1 , product estimate (3.5), and (3.8)-(3.11), we can easily get (3.17)(3.21).

\subsection{Basic energy estimates}

This subsection is devoted to establishing the energy estimates of $(\eta, u)$, which include the zero estimate of $(\eta, u)$ (see Lemma 3.3), the estimates of temporal derivative of $u$ (see Lemma 3.4), the Stokes estimates of $u$ (see Lemma 3.5), the hybrid derivative estimate of $(\eta, u)$ (see Lemma 3.6), and the equivalent estimate of $\mathcal{E}$ (see Lemma 3.7). Next we will establish those estimates in sequence.

Lemma 3.3 Under assumption (3.1), it holds that

$$
\frac{\mathrm{d}}{\mathrm{d} t} \int\left(\bar{\rho} \eta \cdot u+\frac{\mu}{4}|\mathbb{D} \eta|^{2}\right) \mathrm{d} y+\frac{\kappa}{2}\|\sqrt{\bar{\rho}} \mathbb{D} \eta\|_{0}^{2} \lesssim\left\|\left(\eta_{3}, u\right)\right\|_{0}^{2}+\sqrt{\mathcal{E}} \mathcal{D} .
$$

Proof Multiplying $(1.17)_{2}$ by $\eta$ in $L^{2}$ yields that

$$
\frac{\mathrm{d}}{\mathrm{d} t} \int \bar{\rho} \eta \cdot u \mathrm{~d} y=-\int \operatorname{div} \mathcal{S}(q, u, \eta) \cdot \eta \mathrm{d} y+\int\left(\bar{\rho}|u|^{2}+g \bar{\rho}^{\prime} \eta_{3}^{2}\right) \mathrm{d} y+\int \mathcal{N}^{1} \cdot \eta \mathrm{d} y .
$$

By the integration by parts and the boundary condition, we get

$$
\begin{aligned}
& \frac{\mathrm{d}}{\mathrm{d} t} \int\left(\bar{\rho} \eta \cdot u+\frac{\mu}{2}|\nabla \eta|^{2}\right) \mathrm{d} y+\frac{\kappa}{2}\|\sqrt{\bar{\rho}} \mathbb{D} \eta\|_{0}^{2} \\
& \quad \leq c\left\|\left(\eta_{3}, u\right)\right\|_{0}^{2}+\int q \operatorname{div} \eta \mathrm{d} y+\int \mathcal{N}^{1} \cdot \eta \mathrm{d} y .
\end{aligned}
$$

We define that

$$
\Psi(\eta):=\left(\begin{array}{c}
-\eta_{1}\left(\partial_{2} \eta_{2}+\partial_{3} \eta_{3}\right)+\eta_{1}\left(\partial_{2} \eta_{3} \partial_{3} \eta_{2}-\partial_{2} \eta_{2} \partial_{3} \eta_{3}\right) \\
\eta_{1} \partial_{1} \eta_{2}-\eta_{2} \partial_{3} \eta_{3}+\eta_{1}\left(\partial_{1} \eta_{2} \partial_{3} \eta_{3}-\partial_{1} \eta_{3} \partial_{3} \eta_{2}\right) \\
\eta_{1} \partial_{1} \eta_{3}+\eta_{2} \partial_{2} \eta_{3}+\eta_{1}\left(\partial_{1} \eta_{3} \partial_{2} \eta_{2}-\partial_{1} \eta_{2} \partial_{2} \eta_{3}\right)
\end{array}\right) .
$$

It is easy to check that $\operatorname{div} \eta=\operatorname{div} \Psi(\eta)$ due to $\operatorname{det}(\nabla \eta+I)=1$.

Thus we can estimate that

$$
\int q \operatorname{div} \eta \mathrm{d} y=-\int \Psi(\eta) \cdot \nabla q \mathrm{~d} y \lesssim\|\eta\|_{2}^{2}\|\nabla q\|_{0} .
$$

Consequently, inserting the estimate above into (3.23), then using (3.17) and Young's inequality, we immediately have (3.22).

Lemma 3.4 Under assumption (3.1), it holds that

$$
\begin{aligned}
& \frac{\mathrm{d}}{\mathrm{d} t}\left(\left\|\sqrt{\bar{\rho}} u_{t}\right\|_{0}^{2}+\frac{\kappa}{2}\|\sqrt{\bar{\rho}} \mathbb{D} u\|_{0}^{2}-2 \int \nabla q \cdot\left(\mathcal{A}_{t}^{\mathrm{T}} u\right) \mathrm{d} y\right)+c\left\|u_{t}\right\|_{1}^{2} \lesssim\left\|u_{3}\right\|_{0}^{2}+\sqrt{\mathcal{E}} \mathcal{D}, \\
& \frac{\mathrm{d}}{\mathrm{d} t}\left(\frac{\mu}{4}\left\|\nabla_{\mathcal{A}} u_{t}\right\|_{0}^{2}+\int \kappa \bar{\rho} \mathbb{D} u: \nabla u_{t} \mathrm{~d} y\right)+c\left\|u_{t t}\right\|_{0}^{2} \lesssim\left\|u_{t}\right\|_{0}^{2}+\left\|u_{3}\right\|_{0}^{2}+\sqrt{\mathcal{E}} \mathcal{D} .
\end{aligned}
$$


Proof (1) Applying $\partial_{t}$ to $(1.17)_{2}-(1.17)_{4}$ and using $(1.17)_{1}$, one has

$$
\begin{cases}\bar{\rho} u_{t t}+\nabla_{\mathcal{A}} q_{t}-\mu \Delta_{\mathcal{A}} u_{t}-\kappa \operatorname{div}(\bar{\rho} \mathbb{D} u)=g \bar{\rho}^{\prime} u_{3} e_{3}+\mathcal{N}^{2} & \text { in } \Omega, \\ \operatorname{div}_{\mathcal{A}} u_{t}=-\operatorname{div}_{\mathcal{A}_{t}} u & \text { in } \Omega, \\ u_{t}=0 & \text { on } \partial \Omega .\end{cases}
$$

Exploiting integral by parts and $(3.26)_{2}$, we have that

$$
\int q_{t} \operatorname{div} \mathcal{A}_{t} u \mathrm{~d} y=-\frac{\mathrm{d}}{\mathrm{d} t} \int \nabla q \cdot\left(\mathcal{A}_{t}^{\mathrm{T}} u\right) \mathrm{d} y+\int \nabla q \cdot \partial_{t}\left(\mathcal{A}_{t}^{\mathrm{T}} u\right) \mathrm{d} y .
$$

Then, multiplying $(3.26)_{1}$ by $u_{t}$ in $L^{2}$ and using the integration by parts, $(3.26)_{2}-(3.26)_{3}$, and (3.27), we can compute out that

$$
\begin{aligned}
\frac{1}{2} & \frac{\mathrm{d}}{\mathrm{d} t}\left(\left\|\sqrt{\bar{\rho}} u_{t}\right\|_{0}^{2}+\frac{\kappa}{2}\|\sqrt{\bar{\rho}} \mathbb{D} u\|_{0}^{2}-2 \int \nabla q \cdot\left(\mathcal{A}_{t}^{\mathrm{T}} u\right) \mathrm{d} y\right)+\frac{\mu}{2}\left\|\nabla_{\mathcal{A}} u_{t}\right\|_{0}^{2} \\
& =\int\left(g \bar{\rho}^{\prime} u_{3} \partial_{t} u_{3}+\mathcal{N}^{2} \cdot u_{t}-\nabla q \cdot \partial_{t}\left(\mathcal{A}_{t}^{\mathrm{T}} u\right)\right) \mathrm{d} y
\end{aligned}
$$

Accordingly, using product estimate (3.5), (3.15), (3.20), and Young's inequality, we get (3.24).

(2) Similarly to (1.11), we have

$\operatorname{div}_{\mathcal{A}} u_{t t}=-\partial_{t} \operatorname{div}_{\mathcal{A}_{t}} u-\operatorname{div} \mathcal{A}_{t} u_{t}=-\operatorname{div}\left(\mathcal{A}_{t t}^{\mathrm{T}} u+2 \mathcal{A}_{t}^{\mathrm{T}} u_{t}\right)$

Multiplying (3.26) by $u_{t t}$ in $L^{2}$ and using the integration by parts, we can compute out that

$$
\begin{aligned}
& \frac{\mathrm{d}}{\mathrm{d} t}\left(\frac{\mu}{4}\left\|\nabla_{\mathcal{A}} u_{t}\right\|_{0}^{2}+\int \kappa \bar{\rho} \mathbb{D} u: \nabla u_{t} \mathrm{~d} y\right)+\left\|\sqrt{\bar{\rho}} u_{t t}\right\|_{0}^{2} \\
& \quad=\int\left(\kappa \bar{\rho} \mathbb{D} u_{t}: \nabla u_{t}+\nabla q_{t} \cdot\left(\mathcal{A}_{t t}^{\mathrm{T}} u+2 \mathcal{A}_{t}^{\mathrm{T}} u_{t}\right)+g \bar{\rho}^{\prime} u_{3} \partial_{t}^{2} u_{3}+\mathcal{N}^{2} \cdot u_{t t}\right) \mathrm{d} y .
\end{aligned}
$$

Consequently, making use of product estimate (3.5), (3.20), and Young's inequality, we arrive at (3.25).

Lemma 3.5 Under assumption (3.1), we have

$$
\begin{aligned}
& \|(u, q)\|_{\mathrm{S}, i} \lesssim\|\eta\|_{i+2}+\left\|u_{t}\right\|_{i} \quad \text { for } 0 \leq i \leq 1, \\
& \left\|\partial_{t}(u, q)\right\|_{\mathrm{S}, 0} \lesssim\|u\|_{2}+\left\|u_{t t}\right\|_{0}+\sqrt{\mathcal{E} \mathcal{D}} .
\end{aligned}
$$

Proof We apply $\partial_{t}^{j}$ to $(1.17)_{2}-(1.17)_{6}$ for $j=0$ and 1 , and then rewrite the resulting identities as the standard Stokes problem:

$$
\begin{cases}\nabla \partial_{t}^{j} q-\mu \Delta \partial_{t}^{j} u=\partial_{t}^{j} \mathcal{M}^{1} & \text { in } \Omega, \\ \operatorname{div} \partial_{t}^{j} u=\operatorname{div} \partial_{t}^{j} u & \text { in } \Omega, \\ \partial_{t}^{j} u=0 & \text { on } \partial \Omega,\end{cases}
$$


where we have defined that

$$
\mathcal{M}^{1}:=\kappa \operatorname{div}(\bar{\rho} \mathbb{D} \eta)+g \bar{\rho}^{\prime} \eta_{3} e_{3}-\bar{\rho} u_{t}+\mathcal{N}^{1} .
$$

Applying the Stokes estimate to (3.32), we have

$$
\begin{aligned}
& \|(u, q)\|_{\mathrm{S}, i} \lesssim\left\|\mathcal{M}^{1}\right\|_{i}+\|\operatorname{div} u\|_{i+1} \quad \text { for } 0 \leq i \leq 1, \\
& \left\|\partial_{t}(u, q)\right\|_{\mathrm{S}, 0} \lesssim\left\|\mathcal{M}_{t}^{1}\right\|_{0}+\left\|\operatorname{div} u_{t}\right\|_{1} .
\end{aligned}
$$

Using (3.13) and (3.14), we can estimate that

$$
\begin{aligned}
& \left\|\mathcal{M}^{1}\right\|_{i}+\|\operatorname{div} u\|_{i+1} \lesssim\|\eta\|_{i+2}+\left\|u_{t}\right\|_{i}+\|\eta\|_{3}\|u\|_{i+2}+\left\|\mathcal{N}^{1}\right\|_{i}, \\
& \left\|\mathcal{M}_{t}^{1}\right\|_{0}+\left\|\operatorname{div} u_{t}\right\|_{1} \lesssim\|u\|_{2}+\left\|u_{t t}\right\|_{0}+\|\eta\|_{3}\left\|u_{t}\right\|_{2}+\left\|\mathcal{N}_{t}^{1}\right\|_{0} .
\end{aligned}
$$

Putting the above two estimates into (3.34) and (3.35), and then using (3.17) and (3.19), we get (3.30) and (3.31).

Lemma 3.6 Let $(\eta, u)$ satisfy assumption (3.1), the following estimate holds:

$$
\frac{\mathrm{d}}{\mathrm{d} t}{\overline{\|\eta\|_{4}}}^{2}+\|(\eta, u)\|_{4}^{2}+\|q\|_{3}^{2} \lesssim\|\eta\|_{1}^{2}+\left\|u_{t}\right\|_{2}^{2}
$$

where the norm ${\overline{\|\eta\|_{4}}}_{4}$ is equivalent to $\|\eta\|_{4}$.

Proof We shall rewrite (3.32) as a (steady) Stokes problem:

$$
\begin{cases}\nabla q-\Delta \omega=\mathcal{M}^{2} & \text { in } \Omega, \\ \operatorname{div} \omega=\kappa \bar{\rho}^{\prime} \eta_{3}+\mathcal{N}^{3} & \text { in } \Omega, \\ \omega=0 & \text { on } \partial \Omega,\end{cases}
$$

where we have defined that

$$
\mathcal{M}^{2}:=-\bar{\rho} u_{t}+g \bar{\rho}^{\prime} \eta_{3} e_{3}+\kappa \bar{\rho} \nabla \operatorname{div} \eta+\kappa\left(\bar{\rho}^{\prime} \nabla \eta_{3}-\bar{\rho}^{\prime} \partial_{3} \eta-\bar{\rho}^{\prime \prime} \eta\right)+\mathcal{N}^{1}, \quad \omega:=\mu u+\kappa \bar{\rho} \eta .
$$

Note that $\mathcal{N}^{3}$ satisfies

$$
\int\left(\kappa \bar{\rho}^{\prime} \eta_{3}+\mathcal{N}^{3}\right) \mathrm{d} y=0
$$

Applying the Stokes estimate to (3.37), we have, for $0 \leq k \leq 2$,

$$
\|\omega\|_{k+2}^{2}+\|q\|_{k+1}^{2} \lesssim\left\|\eta_{3}\right\|_{k+1}^{2}+\left\|\mathcal{M}^{2}\right\|_{k}^{2}+\left\|\mathcal{N}^{3}\right\|_{k+1}^{2} .
$$

Moreover, by $(1.17)_{1}$, we easily see that

$$
\|\omega\|_{k+2}^{2}=\frac{\mathrm{d}}{\mathrm{d} t}\|\sqrt{\kappa \bar{\rho} \mu} \eta\|_{k+2}^{2}+\|\kappa \bar{\rho} \eta\|_{k+2}^{2}+\|\mu u\|_{k+2}^{2} .
$$


Using (3.12), we can estimate that

$$
\left\|\mathcal{M}^{2}\right\|_{2} \lesssim\|\eta\|_{3}+\left\|u_{t}\right\|_{2}+\|\eta\|_{4}^{2}+\left\|\mathcal{N}^{1}\right\|_{2} .
$$

Consequently, exploiting interpolation inequality (3.4), (3.39), and (3.40), we can derive from (3.38) that

$$
\begin{aligned}
& \frac{\mathrm{d}}{\mathrm{d} t}{\overline{\|\eta\|_{4}}}^{2}+\|\kappa \bar{\rho} \eta\|_{4}^{2}+\|\mu u\|_{4}^{2}+\|q\|_{3}^{2} \\
& \quad \lesssim\|\eta\|_{1}^{2}+\left\|u_{t}\right\|_{2}^{2}+\left\|\mathcal{N}^{1}\right\|_{2}^{2}+\left\|\mathcal{N}^{3}\right\|_{3}^{2},
\end{aligned}
$$

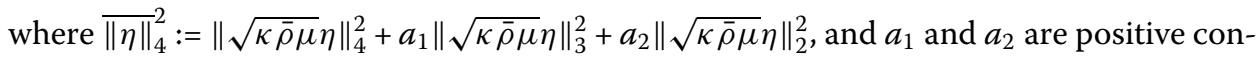
stants. We get (3.36) by inserting (3.17) and (3.21) into (3.41).

Lemma 3.7 Under assumption (3.1), we have

$$
\mathcal{E} \text { is equivalent to }\|\eta\|_{4}^{2}+\|u\|_{3}^{2} .
$$

Proof By (3.30), we see that

$$
\|\eta\|_{4}^{2}+\|u\|_{3}^{2} \lesssim \mathcal{E} \lesssim\|\eta\|_{4}^{2}+\|u\|_{3}^{2}+\left\|u_{t}\right\|_{1}^{2}
$$

To get (3.42), it suffices to verify

$$
\left\|u_{t}\right\|_{1}^{2} \lesssim\|\eta\|_{4}^{2}+\|u\|_{3}^{2}
$$

On the one hand, applying $\partial_{i}$ to $(1.17)_{2}$, we get, for $0 \leq i \leq 3$,

$$
\begin{aligned}
\bar{\rho} \partial_{i} u_{t}+\bar{\rho}^{\prime} u_{t}+\nabla \partial_{i} q= & \mu \operatorname{div} \nabla \partial_{i} u+\kappa \bar{\rho} \operatorname{div} \mathbb{D} \partial_{i} \eta+\kappa \bar{\rho}^{\prime} \operatorname{div} \mathbb{D} \eta \\
& +\kappa \bar{\rho}^{\prime \prime}\left(\partial_{3} \eta+\nabla \eta_{3}\right)+g \bar{\rho}^{\prime} \partial_{i} \eta_{3} e_{3}+g \bar{\rho}^{\prime \prime} \eta_{3} e_{3}+\partial_{i} \mathcal{N}^{1} .
\end{aligned}
$$

Multiplying (3.44) by $\partial_{i} u_{t}$ in $L^{2}$ and using the integral by parts, we get

$$
\begin{aligned}
\int \bar{\rho}\left|\partial_{i} u_{t}\right|^{2} \mathrm{~d} y= & \int\left(\mu \operatorname{div} \nabla \partial_{i} u+\kappa \bar{\rho} \operatorname{div} \mathbb{D} \partial_{i} \eta+\kappa \bar{\rho}^{\prime} \operatorname{div} \mathbb{D} \eta+\kappa \bar{\rho}^{\prime \prime}\left(\partial_{3} \eta+\nabla \eta_{3}\right)\right. \\
& \left.+g \bar{\rho}^{\prime} \partial_{i} \eta_{3} e_{3}+g \bar{\rho}^{\prime \prime} \eta_{3} e_{3}+\partial_{i} \mathcal{N}^{1}-\partial_{i}^{2} q \operatorname{div} u_{t}-\int \bar{\rho}^{\prime} u_{t}\right) \cdot \partial_{i} u_{t} \mathrm{~d} y \\
= & I_{1} .
\end{aligned}
$$

Using product estimate (3.5), we can estimate that

$$
\left|I_{1}\right| \lesssim\left(\|(\eta, u)\|_{3}+\left\|\mathcal{N}^{1}\right\|_{1}\right)\left\|u_{t}\right\|_{1}+\|q\|_{2}\left\|\operatorname{div} u_{t}\right\|_{0}+\left\|u_{t}\right\|_{0}\left\|u_{t}\right\|_{1} .
$$


On the other hand, multiplying $(1.17)_{2}$ by $u_{t}$ in $L^{2}$, and then using the integral by parts, we get

$$
\begin{aligned}
\int \bar{\rho}\left|u_{t}\right|^{2} \mathrm{~d} y & =\int\left(\left(\tilde{\mathcal{A}}^{\mathrm{T}} u_{t}+\tilde{\mathcal{A}}_{t}^{\mathrm{T}} u\right) \cdot \nabla q-\left(\operatorname{div} \mathcal{S}(0, u, \eta)+g \bar{\rho}^{\prime} \eta_{3} e_{3}+\mathcal{N}^{1}\right) \cdot u_{t}\right) \mathrm{d} y \\
& =: I_{2}
\end{aligned}
$$

Making use of (3.11) and product estimate (3.5), we can estimate that

$$
\left|I_{2}\right| \lesssim\left\|u_{t}\right\|_{0}\left(\|(\eta, u)\|_{2}+\|\eta\|_{3}\|\nabla q\|_{0}+\left\|\mathcal{N}^{1}\right\|_{0}\right)+\|u\|_{2}\|u\|_{1}\|\nabla q\|_{0}
$$

Putting the above estimate into (3.47), and then using Young's inequality, (3.17), and (3.30), we get

$$
\left\|u_{t}\right\|_{0}^{2} \lesssim\|\eta\|_{3}^{2}+\|u\|_{2}^{2}
$$

Consequently, inserting (3.49) into (3.46), we can further deduce that

$$
\left|I_{1}\right| \lesssim\left(\|(\eta, u)\|_{3}+\left\|\mathcal{N}^{1}\right\|_{1}\right)\left\|u_{t}\right\|_{1}+\|q\|_{2}\left\|\operatorname{div} u_{t}\right\|_{0}
$$

Finally, inserting (3.50) into (3.45), exploiting (3.14), (3.17), (3.30), and Young's inequality, we immediately get (3.42). This completes the proof of Lemma 3.7 .

\subsection{Gronwall-type energy inequality}

With the estimates of $(\eta, u)$ in Lemmas 3.3-3.7, we can derive a priori Gronwall-type energy inequality as follows.

Lemma 3.8 There exist an energy functional $\mathfrak{E}(t)$ and constants $\delta_{1} \in(0,1)$ and $C_{1}>0$ such that, for any $\delta \leq \delta_{1}$, if the solution $(\eta, u)$ of the transformed VRT problem satisfies (3.1), then $(\eta, u)$ satisfies the Gronwall-type energy inequality

$$
\mathfrak{E}(t)+\int_{0}^{t} \mathcal{D}(\tau) \mathrm{d} \tau \leq C_{1}\left(\left\|\eta^{0}\right\|_{4}^{2}+\left\|u^{0}\right\|_{3}^{2}+\int_{0}^{t}\|(\eta, u)\|_{0}^{2} \mathrm{~d} \tau\right)
$$

and the equivalent estimate

$$
\mathcal{E}(t) \leq C_{1} \mathfrak{E}(t) \lesssim \mathcal{E}(t)
$$

for any $t \in(0, T)$, where the above constants $\delta_{1}$ and $C_{1}$ depend on the domain $\Omega$ and parameters in the transformed VRT problem.

Proof By Lemmas 3.3-3.7, and then making use of (3.15), Young's and interpolation inequalities, we can deduce that there exist constants $c, c_{1}$, and $\tilde{\delta}_{1}$, such that, for any $\delta \leq \tilde{\delta}_{1}$,

$$
\frac{\mathrm{d}}{\mathrm{d} t} \tilde{\mathfrak{E}}+c \mathcal{D} \leq c_{1}^{2}\left(\|(\eta, u)\|_{0}^{2}+\sqrt{\mathcal{E}} \mathcal{D}\right) / c,
$$


where we have defined that

$$
\begin{aligned}
\tilde{\mathfrak{E}}:= & \left(\int \bar{\rho} \eta \cdot u \mathrm{~d} y+\frac{\mu}{4}\|\mathbb{D} \eta\|_{0}^{2}\right)+c_{1}\left(\frac{\mu}{4}\left\|\nabla_{\mathcal{A}} u_{t}\right\|_{0}^{2}+\int \kappa \bar{\rho} \mathbb{D} u: \nabla u_{t} \mathrm{~d} y\right) \\
& +c_{1}^{2}\left(\left\|\sqrt{\bar{\rho}} u_{t}\right\|_{0}^{2}+\frac{\kappa}{2}\|\sqrt{\bar{\rho}} \mathbb{D} u\|_{0}^{2}-2 \int \nabla q \cdot\left(\mathcal{A}_{t}^{\mathrm{T}} u\right) \mathrm{d} y\right)+{\overline{\|\eta\|_{4}^{2}}}^{2} .
\end{aligned}
$$

It is easy to check that

$$
\|\eta\|_{4}^{2}+\|(u, q)\|_{\mathrm{S}, 1}^{2}+\left\|u_{t}\right\|_{1}^{2} \lesssim \tilde{\mathfrak{E}} \lesssim\|\eta\|_{4}^{2}+\|u\|_{1}^{2}+\left\|u_{t}\right\|_{1}^{2} .
$$

Exploiting (3.42) again, there exists a constant $\tilde{\delta}_{2} \leq \tilde{\delta}_{1}$ such that, for any $\delta \leq \tilde{\delta}_{2}$, we further deduce from (3.53) that

$$
\frac{\mathrm{d}}{\mathrm{d} t} \tilde{\mathfrak{E}}+c \mathcal{D} \leq\|(\eta, u)\|_{0}^{2} / c .
$$

Integrating the above inequality with respect to $t$ and using (3.42) with $t=0$, we get (3.51) by taking $\delta_{1}:=\tilde{\delta}_{2}$ and $\mathfrak{E}=\tilde{\mathfrak{E}} / c$ for some constant $c$.

We can establish the existence of unique classical solution of the transformed VRT problem by using the standard iteration scheme as in [23, Proposition 3.1]. In addition, the solution enjoys the Gronwall-type energy inequality. So, we have the following conclusion.

\section{Proposition 3.1}

(1) Let $\left(\eta^{0}, u^{0}\right) \in H_{0}^{4} \times H_{0}^{3}$ and $\zeta^{0}:=\eta^{0}+y$. There exists sufficiently small $\delta_{2} \in(0,1)$ such that, if $\left(\eta^{0}, u^{0}\right)$ satisfies

$$
\sqrt{\left\|\eta^{0}\right\|_{4}^{2}+\left\|u^{0}\right\|_{3}^{2}}<\delta_{2}
$$

and

$$
2 \operatorname{det} \nabla \zeta^{0}=1, \quad \operatorname{div}_{\mathcal{A}^{0}} u^{0}=0 \quad \text { in } \Omega,
$$

then there are a local existence time $T^{\max }>0$, depending on $\delta_{2}$, the domain, and the known parameters in the transformed VRT problem, and a unique local-in-time classical solution $(\eta, u) \in C^{0}\left(\left[0, T^{\max }\right), H^{4} \times H^{3}\right)$ with a unique (up to a constant) associated pressure $q$ to the transformed VRT problem, where the solution enjoys the regularity (1.23)-(1.24) with $T^{\max }$ in place of $T$.

(2) In addition, if $(\eta, u)$ satisfies

$$
\sup _{t \in[0, T)} \sqrt{\|\eta(t)\|_{4}^{2}+\|u(t)\|_{3}^{2}} \leq \delta_{1} \quad \text { for some } T<T^{\max }
$$

then the solution $(\eta, u)$ enjoys the Gronwall-type energy inequality (3.51) and the equivalent estimate (3.52) on $(0, T)$. 
Remark 3.1 The initial data of the associated pressure $q$ constructed in Proposition 3.1 is the weak solution of the boundary value problem

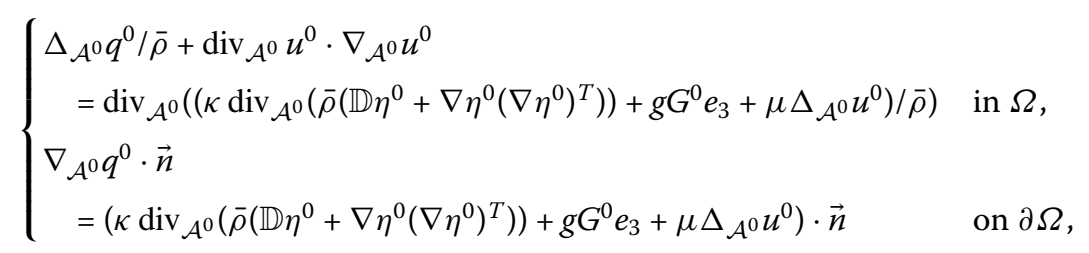

where $\vec{n}$ denotes the unit normal. It should be noted that test functions for the weak form of (3.56) ${ }_{1}$ belong to $H^{1}$ (referring to (3.64) in [25]). Since there exists $\delta_{3}$ such that, for any $\|\eta\|_{3} \leq \delta_{3}$,

$$
\|\nabla q\|_{0} \leq\left\|\nabla_{\mathcal{A}} q\right\|_{0} \quad \text { and } \quad \vec{n} \neq 0
$$

thus we easily see that, for any $\left\|\eta^{0}\right\|_{3} \leq \delta_{3}$, the weak solution $q^{0} \in H^{1}$ of (3.56) is unique up to a constant for given $\left(\eta^{0}, u^{0}\right)$.

Remark 3.2 Note that, for any classical solution $(\eta, u)$ with an associated pressure $q$ constructed by Proposition 3.1, for any $t_{0} \in\left(0, T^{\max }\right),\left.(\eta, u)\right|_{t=t_{0}}$ automatically satisfies (3.55) with $\left.(\eta, u)\right|_{t=t_{0}}$ in place of $\left(\eta^{0}, u^{0}\right)$. If we consider $\left.(\eta, u)\right|_{t=t_{0}}$ as a new initial data, then the new initial data can define a unique local-in-time classical solution $(\tilde{\eta}, \tilde{u})$ constructed by Proposition 3.1.

\section{Construction of initial data for nonlinear solutions}

For any given $\delta>0$, let

$$
\left(\eta^{\mathrm{a}}, u^{\mathrm{a}}, q^{\mathrm{a}}\right)=\delta e^{\Lambda t}\left(\tilde{\eta}^{0}, \tilde{u}^{0}, \tilde{q}^{0}\right)
$$

where $\left(\tilde{\eta}^{0}, \tilde{u}^{0}, \tilde{q}^{0}\right):=(w / \Lambda, w, \beta)$ and $(w, \beta) \in\left(\mathcal{A} \cap H^{\infty}\right) \times\left(\underline{H}^{1} \cap H^{\infty}\right)$ comes from Proposition 2.1. Then $\left(\eta^{\mathrm{a}}, u^{\mathrm{a}}\right)$ is a solution to the linearized VRT problem and enjoys the estimates, for any $i, j \geq 0$,

$$
\left\|\partial_{t}^{i}\left(\eta^{\mathrm{a}}, u^{\mathrm{a}}, q^{\mathrm{a}}\right)\right\|_{\mathrm{S}, j} \leq c(i, j) \delta e^{\Lambda t}
$$

Moreover, by (2.3), for $\tilde{\chi}^{0}:=\tilde{\eta}^{0}$ or $\tilde{u}^{0}$,

$$
\left\|\tilde{\chi}_{\mathrm{h}}^{0}\right\|_{L^{1}}\left\|\tilde{\chi}_{3}^{0}\right\|_{L^{1}}>0
$$

Next we modify the initial data of the linear solution $\left(\eta^{\mathrm{a}}, u^{\mathrm{a}}, q^{\mathrm{a}}\right)$, so that the obtained new initial data satisfy the necessary compatibility conditions (3.55) and (3.56).

Proposition 4.1 Let $\left(\tilde{\eta}^{0}, \tilde{u}^{0}, \tilde{q}^{0}\right)$ be the same as in (4.1), then there exist a constant $\delta_{4}$ (depending on $\left(\tilde{\eta}^{0}, \tilde{u}^{0}, \tilde{q}^{0}\right)$, the domain, and the parameters in the transformed VRT problem) such that, for any $\delta \in\left(0, \delta_{4}\right)$, there is a couple $\left(\eta^{\mathrm{r}}, u^{\mathrm{r}}, q^{\mathrm{r}}\right) \in H_{0}^{4} \times H_{0}^{3} \times H^{2}$ enjoying the following properties: 
(1) The modified initial data

$$
\left(\eta_{0}^{\delta}, u_{0}^{\delta}, q_{0}^{\delta}\right):=\delta\left(\tilde{\eta}^{0}, \tilde{u}^{0}, \tilde{q}^{0}\right)+\delta^{2}\left(\eta^{\mathrm{r}}, u^{\mathrm{r}}, q^{\mathrm{r}}\right)
$$

belongs to $H_{0}^{4} \times H_{0}^{3} \times H^{2}$ and satisfies (3.55) and (3.56) with $\left(\eta_{0}^{\delta}, u_{0}^{\delta}, q_{0}^{\delta}\right)$ in place of $\left(\eta^{0}, u^{0}, q^{0}\right)$.

(2) Uniform estimate:

$$
\sqrt{\left\|\eta^{\mathrm{r}}\right\|_{4}^{2}+\left\|\left(u^{\mathrm{r}}, q^{\mathrm{r}}\right)\right\|_{\mathrm{S}, 1}^{2}} \leq C_{2}
$$

where the constant $C_{2} \geq 1$ depends on the domain and the known parameters, but is independent of $\delta$.

Proof We rewrite $\left(\eta_{0}^{\delta}, u_{0}^{\delta}, q_{0}^{\delta}, \tilde{\eta}^{0}, \tilde{u}^{0}, \tilde{q}^{0}\right)$ by $\left(\eta^{\delta}, u^{\delta}, q^{\delta}, \tilde{\eta}, \tilde{u}, \tilde{q}\right)$ for simplicity.

Recalling the construction of $(\tilde{\eta}, \tilde{u}, \tilde{q})$, we can see that $(\tilde{\eta}, \tilde{u}, \tilde{q})$ satisfies

$$
\begin{cases}\operatorname{div} \tilde{\eta}=\operatorname{div} \tilde{u}=0 & \text { in } \Omega, \\ \Lambda \bar{\rho} \tilde{u}+\operatorname{div} \mathcal{S}(\tilde{q}, \tilde{u}, \tilde{\eta})=g \bar{\rho}^{\prime} \tilde{\eta} e_{3} & \text { in } \Omega, \\ (\tilde{\eta}, \tilde{u})=0 & \text { on } \partial \Omega .\end{cases}
$$

If $\left(\eta^{\mathrm{r}}, u^{\mathrm{r}}, q^{\mathrm{r}}\right) \in H_{0}^{4} \times H_{0}^{3} \times H^{2}$ satisfies

$$
\begin{cases}\operatorname{div} \eta^{\mathrm{r}}=O\left(\eta^{\mathrm{r}}\right), \quad \operatorname{div} u^{\mathrm{r}}=-\operatorname{div}_{\mathcal{A}^{\delta}}\left(\tilde{u}+\delta u^{\mathrm{r}}\right) / \delta, & \\ \operatorname{div}_{\mathcal{A}^{\delta}} \Upsilon=\delta \operatorname{div}_{\mathcal{\mathcal { A }}^{\delta}} \tilde{u} & \text { in } \Omega, \\ \operatorname{div} \mathcal{S}\left(q^{\mathrm{r}}, u^{\mathrm{r}}, \eta^{\mathrm{r}}\right)-g \bar{\rho}^{\prime} \eta_{3}^{\mathrm{r}} e_{3} & \\ \quad=\left(\mathcal{N}^{1}\left(\eta^{\delta}, u^{\delta}, q^{\delta}\right)+\Lambda \bar{\rho} \Upsilon-\bar{\rho} u^{\delta} \cdot \nabla_{\mathcal{A}^{\delta}} u^{\delta}\right) / \delta^{2} & \text { in } \Omega, \\ \left(\eta^{\mathrm{r}}, u^{\mathrm{r}}, \Upsilon\right)=0 & \text { on } \partial \Omega,\end{cases}
$$

where $\left(\eta^{\delta}, u^{\delta}, q^{\delta}\right)$ is given in the mode $(4.4), \zeta^{\delta}:=\eta^{\delta}+y, \mathcal{A}^{\delta}:=\left(\nabla \zeta^{\delta}\right)^{-1}, \tilde{\mathcal{A}}^{\delta}:=\mathcal{A}^{\delta}-I$ and $O\left(\eta^{\mathrm{r}}\right):=\delta^{-2} \operatorname{div} \Psi\left(\delta \tilde{\eta}+\delta^{2} \eta^{\mathrm{r}}\right)$. Then, by (4.6), it is easy to check that $\left(\eta^{\delta}, u^{\delta}, q^{\delta}\right)$ belongs to $H_{0}^{4} \times H_{0}^{3} \times H^{2}$ and satisfies (3.55) and (3.56) with $\left(\eta^{\delta}, u^{\delta}, q^{\delta}\right)$ in place of $\left(\eta^{0}, u^{0}, q^{0}\right)$. In fact, $\left(\eta^{\delta}, u^{\delta}, q^{\delta}\right)$ satisfies

$$
\begin{aligned}
& \Lambda \delta \tilde{u}+u^{\delta} \cdot \nabla_{\mathcal{A}^{\delta}} u^{\delta} \\
& \quad+\left(\nabla_{\mathcal{A}^{\delta}} q^{\delta}-\kappa \operatorname{div}_{\mathcal{A}^{\delta}}\left(\bar{\rho}\left(\mathbb{D} \eta^{\delta}+\nabla \eta^{\delta}\left(\nabla \eta^{\delta}\right)^{T}\right)\right)-g G^{\delta} e_{3}-\mu \Delta_{\mathcal{A}^{\delta}} u^{\delta}\right) / \bar{\rho}=\Lambda \Upsilon \quad \text { in } \Omega,
\end{aligned}
$$

where $G^{\delta}=\bar{\rho}\left(y_{3}+\eta_{3}^{\delta}\right)-\bar{\rho}\left(y_{3}\right)$, which automatically implies (3.56). Next we construct such $\left(\eta^{\mathrm{r}}, u^{\mathrm{r}}, q^{\mathrm{r}}\right)$ enjoying (4.7).

(1) To construct $\eta^{\mathrm{r}}$, we consider a Stokes problem, for a given function $\xi \in H_{0}^{4}(\Omega)$,

$$
\begin{cases}\nabla \varpi-\mu \Delta \eta=0, & \operatorname{div} \eta=O(\xi) \\ \eta=0 & \text { in } \Omega,\end{cases}
$$


where $O(\xi):=\delta^{-2} \operatorname{div} \Psi\left(\delta \tilde{\eta}+\delta^{2} \xi\right)$. Using product estimate (3.5), we can estimate that

$$
\|O(\xi)\|_{3} \leq c\left(1+\delta\|\xi\|_{4}+\delta^{2}\|\xi\|_{4}^{2}+\delta^{3}\|\xi\|_{4}^{3}\right) \leq c_{1}\left(1+\delta^{3}\|\xi\|_{4}^{3}\right) .
$$

By virtue of $\int O(\xi) \mathrm{d} y=0$ and the existence theory of Stokes problem, we can derive that there exists a solution $(\eta, \varpi) \in H_{0}^{4} \times \underline{H}^{3}$ of (4.8). In addition, it holds that

$$
\|(\eta, \varpi)\|_{S, 2} \leq\|O(\xi)\|_{3} \leq c_{1}\left(1+\delta^{3}\|\xi\|_{4}^{3}\right),
$$

where the letter $c_{1}$ denotes a fixed constant dependent on $\delta$.

Now, we construct an approximate function sequence $\left\{\left(\eta^{n}, \varpi^{n}\right)\right\}_{n=1}^{\infty}$ such that, for any $n \geq 2$,

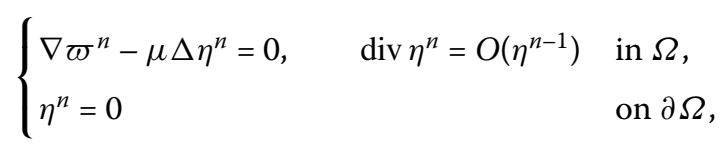

where $\eta \in H_{0}^{4}$ and $\left\|\eta^{1}\right\|_{4} \leq 2 c_{1}$. From (4.9) we also have, for any $n \geq 2$,

$$
\left\|\left(\eta^{n}, \varpi^{n}\right)\right\|_{\mathrm{S}, 2} \leq c_{1}\left(1+\delta^{3}\left\|\eta^{n-1}\right\|_{4}^{3}\right)
$$

which implies

$$
\left\|\left(\eta^{n}, \varpi^{n}\right)\right\|_{\mathrm{S}, 2} \leq 2 c_{1}
$$

for any $n \geq 2$ and any $\delta \leq 1 / 2 c_{1}$.

We can further show that $\left\{\left(\eta^{n}, \varpi^{n}\right)\right\}_{n=1}^{\infty}$ is a Cauchy sequence. Noting that

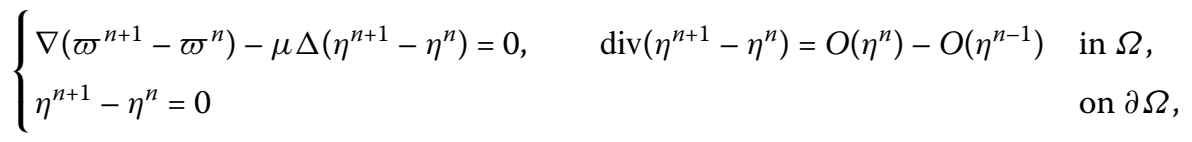

using Stokes estimate, it holds that

$$
\left\|\left(\eta^{n+1}-\eta^{n}, \varpi^{n+1}-\varpi^{n}\right)\right\|_{\mathrm{S}, 2} \leq c\left\|O\left(\eta^{n}\right)-O\left(\eta^{n-1}\right)\right\|_{3} .
$$

Thus, making use of (4.11) and product estimate (3.5), we get

$$
\left\|O\left(\eta^{n}\right)-O\left(\eta^{n-1}\right)\right\|_{3} \leq c \delta\left\|\eta^{n}-\eta^{n-1}\right\|_{4} .
$$

Inserting the above inequality into (4.12) and taking $\delta$ appropriately small and that $\left\{\left(\eta^{n}, \varpi^{n}\right)\right\}_{n=1}^{\infty}$ is a Cauchy sequence in $H^{4} \times H^{3}$, for some limit function $\eta^{\mathrm{r}}$,

$$
\left(\eta^{n}, \varpi^{n}\right) \rightarrow\left(\eta^{\mathrm{r}}, \varpi^{\mathrm{r}}\right) \quad \text { in } H_{0}^{4} \times H^{3} .
$$

Accordingly, we can take to the limit in (4.10) as $n \rightarrow \infty$ to see that the limit function $\left(\eta^{\mathrm{r}}, \varpi^{\mathrm{r}}\right)$ satisfies

$$
\left\{\begin{array}{lll}
\nabla \varpi^{\mathrm{r}}-\mu \Delta \eta^{\mathrm{r}}=0, & \operatorname{div} \eta^{\mathrm{r}}=O\left(\eta^{\mathrm{r}}\right) & \text { in } \Omega \\
\eta^{\mathrm{r}}=0 & \text { on } \partial \Omega .
\end{array}\right.
$$


So $\eta^{\delta}$ satisfies $\operatorname{det}\left(\nabla \eta^{\delta}+I\right)=1$ for appropriately small $\delta$. Moreover, by (4.11), we have

$$
\left\|\eta^{\mathrm{r}}\right\|_{4} \leq 2 c_{1}
$$

(2) Noting that $\left\|\tilde{\mathcal{A}}^{\delta}\right\|_{3} \lesssim\left\|\eta^{\delta}\right\|_{4}$ for sufficiently small $\delta$, where $\eta^{r}$ is constructed in step (1), thus, following the construction of $\eta^{r}$, for sufficiently small $\delta$, we can easily get a solution $(\Upsilon, \phi)$ of

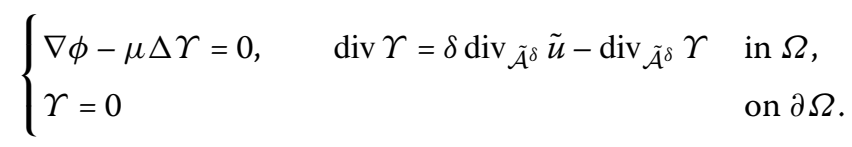

Moreover, by the Stokes estimate,

$$
\|(\Upsilon, \phi)\|_{\mathrm{S}, 2} \lesssim\left\|\delta \operatorname{div}_{\tilde{\mathcal{A}}^{\delta}} \tilde{u}-\operatorname{div}_{\tilde{\mathcal{A}}^{\delta}} \Upsilon\right\|_{3} \lesssim c\left(\delta^{2}+\delta\|\Upsilon\|_{4}\right),
$$

where the constant $c$ is independent of $\delta$. The above estimate implies, for sufficiently small $\delta$,

$$
\|(\Upsilon, \phi)\|_{\mathrm{S}, 2} \lesssim c \delta^{2}
$$

(3) Following the construct of $\eta^{\mathrm{r}}$ as in (1), we can also construct $\left(u^{\mathrm{r}}, q^{\mathrm{r}}\right)$. We consider the following Stokes problem for a given function $(w, p) \in H_{0}^{3} \times H^{2}$ :

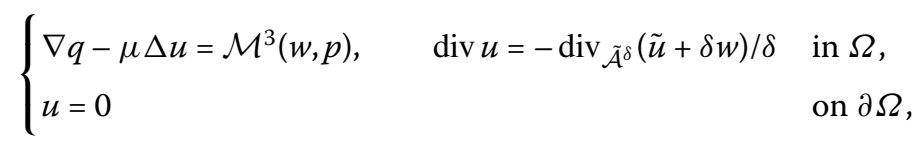

where $\eta^{\mathrm{r}}$ is provided by (4.13), and we have defined that

$$
\begin{aligned}
\mathcal{M}^{3}(w, p):= & \kappa \bar{\rho} \mathbb{D} \eta^{r}+g \bar{\rho}^{\prime} \eta_{3}^{r} e_{3}+\left(\mathcal{N}^{1}\left(\delta \tilde{\eta}+\delta^{2} \eta^{r}, \delta \tilde{u}+\delta^{2} w, \delta \tilde{q}+\delta^{2} p\right)\right. \\
& \left.+\Lambda \bar{\rho} \Upsilon-\bar{\rho}\left(\delta \tilde{u}+\delta^{2} w\right) \cdot \nabla_{\mathcal{A}^{\delta}}\left(\delta \tilde{u}+\delta^{2} w\right)\right) / \delta^{2}
\end{aligned}
$$

It is obvious that $-\int \operatorname{div}_{\tilde{\mathcal{A}}^{\delta}}(\tilde{u}+\delta w) / \delta \mathrm{d} y=0$. So, by the existence theory of Stokes problem, we can see that there exists a solution $(u, q) \in H_{0}^{3} \times H^{2}$ to (4.16), and the solution satisfies

$$
\|(u, q)\|_{\mathrm{S}, 1} \lesssim\left\|\mathcal{M}^{3}(w, p)\right\|_{1}+\left\|\operatorname{div}_{\tilde{\mathcal{A}}^{\delta}}(\tilde{u}+\delta w)\right\|_{2} / \delta .
$$

By (3.17), (4.14), (4.15), and Young's inequality, we have, for some constant $c_{2}$,

$$
\|(u, q)\|_{S, 1} \leq c_{2}\left(1+\delta^{2}\left(\|w\|_{3}^{2}+\|p\|_{2}^{2}\right)\right) .
$$

Next, we can construct an approximate function sequence $\left\{\left(u^{n}, q^{n}\right)\right\}_{n=1}^{\infty}$ such that, for any $n \geq 2$,

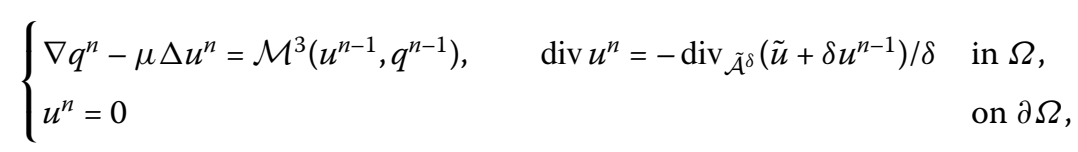


where $u^{1} \in H_{0}^{3}$ and $\left\|\left(u^{1}, q^{1}\right)\right\|_{\mathrm{s}, 1} \leq c_{2}$. By (4.18), we know that

$$
\left\|\left(u^{n}, q^{n}\right)\right\|_{S, 1} \leq c_{2}\left(1+\delta^{2}\left(\left\|u^{n-1}\right\|_{3}^{2}+\left\|q^{n-1}\right\|_{2}^{2}\right)\right)
$$

for any $n \geq 2$, which implies that

$$
\left\|\left(u^{n}, q^{n}\right)\right\|_{\mathrm{S}, 1} \leq 2 c_{2}
$$

for any $n$ and any $\delta \leq 1 / 2 c_{2}$.

We further prove that $\left\{u^{n}, q^{n}\right\}_{n=1}^{\infty}$ is a Cauchy sequence. Noting that

$$
\begin{cases}\nabla\left(q^{n+1}-q^{n}\right)-\mu \Delta\left(u^{n+1}-u^{n}\right)=\mathcal{M}^{3}\left(u^{n}, q^{n}\right)-\mathcal{M}^{3}\left(u^{n-1}, q^{n-1}\right), & \text { in } \Omega, \\ \operatorname{div}\left(u^{n+1}-u^{n}\right)=-\operatorname{div}_{\tilde{\mathcal{A}}^{\delta}}\left(u^{n}-u^{n-1}\right) / \delta & \text { in } \Omega, \\ u^{n+1}-u^{n}=0 & \text { on } \partial \Omega,\end{cases}
$$

exploiting the Stokes estimate, we get

$$
\begin{aligned}
& \left\|\left(u^{n+1}-u^{n}, q^{n+1}-q^{n}\right)\right\|_{\mathrm{S}, 1} \\
& \quad \lesssim\left\|\mathcal{M}^{3}\left(u^{n}, q^{n}\right)-\mathcal{M}^{3}\left(u^{n-1}, q^{n-1}\right)\right\|_{1}+\left\|\operatorname{div}_{\tilde{\mathcal{A}}^{\delta}}\left(u^{n}-u^{n-1}\right)\right\|_{2} .
\end{aligned}
$$

By (3.17) and product estimate (3.5), we further have

$$
\left\|\left(u^{n+1}-u^{n}, q^{n+1}-q^{n}\right)\right\|_{\mathrm{S}, 0} \lesssim c \delta\left\|\left(u^{n}-u^{n-1}, q^{n}-q^{n-1}\right)\right\|_{\mathrm{S}, 0^{\prime}}
$$

which presents that $\left\{\left(u^{n}, q^{n}\right)\right\}_{n=1}^{\infty}$ is a Cauchy sequence in $H_{0}^{3} \times H^{2}$ by choosing sufficiently small $\delta$. Therefore, we can directly get a limit function $\left(u^{\mathrm{r}}, q^{\mathrm{r}}\right) \in H_{0}^{3} \times H^{2}$ as in step (1), which solves

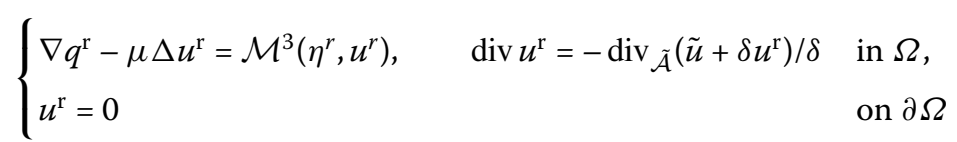

by (4.19). Hence $u^{\delta}$ satisfies $\operatorname{div}_{\mathcal{A}} u^{\delta}=0$ for sufficiently small $\delta$. Moreover, by (4.20), $\left\|\left(u^{\mathrm{r}}, q^{\mathrm{r}}\right)\right\|_{\mathrm{s}, 1} \leq 2 c_{2}$, which, together with (4.14), yields (4.5).

\section{Error estimates}

We define that

$$
\begin{aligned}
& C_{3}:=\sqrt{\left\|\tilde{\eta}^{0}\right\|_{4}^{2}+\left\|\tilde{u}^{0}\right\|_{3}^{2}}+C_{2} \geq 1, \\
& \delta<\delta_{0}:=\min \left\{\delta_{1}, \delta_{2}, \delta_{3}, C_{3} \delta_{4}\right\} / 2 C_{3}<1,
\end{aligned}
$$

and $\left(\eta_{0}^{\delta}, u_{0}^{\delta}\right)$ is constructed by Proposition 4.1. By Proposition 3.1, there exists a (nonlinear) solution $(\eta, u)$ with an associated pressure $q$ of the transformed VRT problem with initial value $\left(\eta_{0}^{\delta}, u_{0}^{\delta}\right)$. To estimate the error between the (nonlinear) solution $(\eta, u)$ and the linear solution $\left(\eta^{\mathrm{a}}, u^{\mathrm{a}}\right)$ provided by $(4.1)$, we define an error function $\left(\eta^{\mathrm{d}}, u^{\mathrm{d}}\right):=(\eta, u)-\left(\eta^{\mathrm{a}}, u^{\mathrm{a}}\right)$. 
Proposition 5.1 Let $(\eta, u)$ be the classical solution of the transformed VRT problem with initial value $\left(\eta_{0}^{\delta}, u_{0}^{\delta}\right)$ with an associated pressure $q$, and $\theta \in(0,1)$ be a small constant such that (3.9)-(3.11) and (3.20) hold for $(\eta, u)$ satisfying (3.1) with $\theta$ in place of $\delta$. For given constants $\gamma$ and $\beta$, if

$$
\begin{aligned}
& \sqrt{\|\eta\|_{4}^{2}+\|u\|_{3}^{2}} \leq \theta, \quad \delta e^{\Lambda t} \leq \beta, \\
& \|\eta(t)\|_{4}^{2}+\|(u(t), q(t))\|_{\mathrm{S}, 1}^{2}+\left\|u_{t}\right\|_{1}^{2} \\
& +\int_{0}^{t}\left(\|(u(\tau), q(\tau))\|_{\mathrm{S}, 2}^{2}+\left\|u_{\tau}\right\|_{2}^{2}\right) \mathrm{d} \tau \leq\left(\gamma \delta e^{\Lambda t}\right)^{2}
\end{aligned}
$$

on some interval $(0, T)$, then there exists a constant $C_{4}$ such that, for any $\delta \in(0,1)$ and any $t \in(0, T)$,

$$
\left\|\chi^{\mathrm{d}}\right\|_{\mathfrak{X}}+\left\|u_{t}^{\mathrm{d}}\right\|_{L^{1}} \leq C_{4} \sqrt{\delta^{3} e^{3 \Lambda t}}
$$

where $\chi=\eta$ or $u, \mathfrak{X}=W^{1,1}$ or $H^{1}$, and $C_{4}$ is independent of $T$.

Proof Subtracting the transformed VRT and the linearized VRT problems, we get

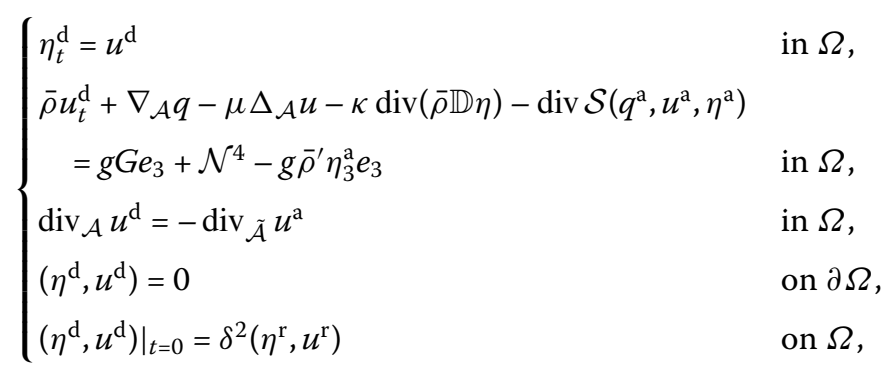

where

$$
\mathcal{N}^{4}:=\kappa\left(\operatorname{div}_{\mathcal{A}}\left(\bar{\rho} \nabla \eta \nabla \eta^{\mathrm{T}}\right)+\operatorname{div}_{\tilde{\mathcal{A}}}(\bar{\rho} \mathbb{D} \eta)\right)
$$

Applying $\partial_{t}$ to $(5.4)_{2}-(5.4)_{4}$ to have

$$
\begin{cases}\bar{\rho} u_{t t}^{\mathrm{d}}+\nabla_{\mathcal{A}} q_{t}^{\mathrm{d}}-\mu \Delta_{\mathcal{A}} u_{t}^{\mathrm{d}}-\kappa \operatorname{div}\left(\bar{\rho} \mathbb{D} u^{\mathrm{d}}\right) & \\ \quad=\mu \operatorname{div} \nabla_{\tilde{\mathcal{A}}} u_{t}^{\mathrm{a}}-\nabla_{\tilde{\mathcal{A}}} q_{t}^{\mathrm{a}}+\mu \operatorname{div}_{\tilde{\mathcal{A}}} \nabla_{\mathcal{A}} u_{t}^{\mathrm{a}}+g \bar{\rho}^{\prime} u_{3}^{\mathrm{d}} e_{3}+\mathcal{N}^{2} & \text { in } \Omega, \\ \operatorname{div}_{\mathcal{A}} u_{t}^{\mathrm{d}}=-\operatorname{div}_{\mathcal{A}_{t}} u-\operatorname{div}_{\tilde{\mathcal{A}}} u_{t}^{\mathrm{a}} & \text { in } \Omega, \\ u_{t}^{\mathrm{d}}=0 & \text { on } \partial \Omega .\end{cases}
$$

Following the argument of (3.28), we can formally infer from (5.5) that

$$
\begin{aligned}
& \frac{1}{2} \frac{\mathrm{d}}{\mathrm{d} t}\left(\left\|\sqrt{\bar{\rho}} u_{t}^{\mathrm{d}}\right\|_{0}^{2}+E\left(u^{\mathrm{d}}\right)-2 \int\left(\nabla q^{\mathrm{d}} \cdot\left(\mathcal{A}_{t}^{\mathrm{T}} u\right)+\nabla q^{\mathrm{d}} \cdot\left(\tilde{\mathcal{A}}^{\mathrm{T}} u_{t}^{\mathrm{a}}\right)\right) \mathrm{d} y\right)+\mu\left\|\nabla_{\mathcal{A}} u_{t}^{\mathrm{d}}\right\|_{0}^{2} \\
&=\int\left(\left(\mu \operatorname{div} \nabla_{\tilde{\mathcal{A}}} u_{t}^{\mathrm{a}}+\mu \operatorname{div}_{\tilde{\mathcal{A}}} \nabla_{\mathcal{A}} u_{t}^{\mathrm{a}}-\nabla_{\tilde{\mathcal{A}}} q_{t}^{\mathrm{a}}+\mathcal{N}^{2}\right) \cdot u_{t}^{\mathrm{d}}\right) \mathrm{d} y \\
& \quad-\int\left(\nabla q^{\mathrm{d}} \cdot \partial_{t}\left(\mathcal{A}_{t}^{\mathrm{T}} u\right)+\nabla q^{\mathrm{d}} \cdot \partial_{t}\left(\tilde{\mathcal{A}}^{\mathrm{T}} u_{t}^{\mathrm{a}}\right)\right) \mathrm{d} y=: R_{1}(t) .
\end{aligned}
$$


Integrating the above identity in time from 0 to $t$ yields that

$$
\begin{aligned}
& \left\|\sqrt{\bar{\rho}} u_{t}^{\mathrm{d}}\right\|_{0}^{2}+E\left(u^{\mathrm{d}}\right)+2 \mu \int_{0}^{t}\left\|\nabla_{\mathcal{A}} u_{\tau}^{\mathrm{d}}\right\|_{0}^{2} \mathrm{~d} \tau \\
& \quad=2\left(\int_{0}^{t} R_{1}(\tau) \mathrm{d} \tau+R_{2}(t)-R_{2}(0)\right)+E\left(\left.u^{\mathrm{d}}\right|_{t=0}\right)+\left.\left\|\sqrt{\bar{\rho}} u_{t}^{\mathrm{d}}\right\|_{0}^{2}\right|_{t=0},
\end{aligned}
$$

where we have defined that

$$
R_{2}(t):=\int\left(\nabla q^{\mathrm{d}} \cdot\left(\mathcal{A}_{t}^{\mathrm{T}} u\right)+\nabla q^{\mathrm{d}} \cdot\left(\tilde{\mathcal{A}}^{\mathrm{T}} u_{t}^{\mathrm{a}}\right)\right) \mathrm{d} y
$$

Noting that $(\eta, u)$ satisfies estimates (3.8)-(3.11) and (3.20), thus, exploiting (4.2), (5.2), and product estimate (3.5), we have

$$
\int_{0}^{t} R_{1}(\tau) \mathrm{d} \tau \lesssim \delta^{3} e^{3 \Lambda t}
$$

Similarly, we can estimate that

$$
R_{2}(t) \lesssim \delta^{3} e^{3 \Lambda t}
$$

which also yields that

$$
R_{2}(0) \lesssim \delta^{3} e^{3 \Lambda t}
$$

Noting that $u^{\mathrm{d}}(0)=\delta^{2} u^{\mathrm{r}}$, we have

$$
E\left(\left.u^{\mathrm{d}}\right|_{t=0}\right) \lesssim \delta^{4}\left\|u^{\mathrm{r}}\right\|_{2}^{2} \lesssim \delta^{3} e^{3 \Lambda t} .
$$

Next we turn to deriving the estimate for $\left.\left\|\sqrt{\rho} u_{t}^{\mathrm{d}}\right\|_{0}^{2}\right|_{t=0}$. Recalling that

$$
\operatorname{div} u_{t}^{\mathrm{d}}=-\operatorname{div} \partial_{t}\left(\tilde{\mathcal{A}}^{\mathrm{T}} u\right)
$$

thus, taking the inner product of $(5.4)_{2}$ and $u_{t}^{\mathrm{d}}$ in $L^{2}$ and using the integration by parts, we have

$$
\begin{aligned}
\int \bar{\rho}\left|u_{t}^{\mathrm{d}}\right|^{2} \mathrm{~d} y= & \int\left(\mu\left(\operatorname{div}_{\tilde{\mathcal{A}}} \nabla_{\mathcal{A}} u+\operatorname{div} \nabla_{\tilde{\mathcal{A}}} u\right)-\nabla_{\tilde{\mathcal{A}}} q-\operatorname{div} \mathcal{S}\left(0, u^{\mathrm{d}}, \eta^{\mathrm{d}}\right)\right. \\
& \left.+g G e_{3}-g \bar{\rho}^{\prime} \eta_{3}^{\mathrm{a}} e_{3}+\mathcal{N}^{4}\right) \cdot u_{t}^{\mathrm{d}} \mathrm{d} y+\int \nabla q^{\mathrm{d}} \cdot \partial_{t}\left(\tilde{\mathcal{A}}^{\mathrm{T}} u\right) \mathrm{d} y .
\end{aligned}
$$

Following the arguments of (3.43), we infer from (5.11) that

$$
\left\|\sqrt{\bar{\rho}} u_{t}^{\mathrm{d}}\right\|_{0}^{2} \lesssim \delta e^{\Lambda t}\left(\delta^{2} e^{2 \Lambda t}+\left\|\eta^{\mathrm{d}}\right\|_{3}+\left\|u^{\mathrm{d}}\right\|_{2}\right),
$$

which, together with the initial data $(5.4)_{5}$, implies that

$$
\left.\left\|\sqrt{\bar{\rho}} u_{t}^{\mathrm{d}}\right\|_{0}^{2}\right|_{t=0} \lesssim \delta^{3} e^{3 \Lambda t}
$$


Accordingly, putting (5.7)-(5.10) and (5.12) into (5.6) yields

$$
\left\|\sqrt{\bar{\rho}} u_{t}^{\mathrm{d}}\right\|_{0}^{2}+2 \mu \int_{0}^{t}\left\|\nabla_{\mathcal{A}} u_{\tau}^{\mathrm{d}}\right\|_{0}^{2} \mathrm{~d} \tau \leq-E\left(u^{\mathrm{d}}\right)+c \delta^{3} e^{3 \Lambda t} .
$$

To deal with $-E\left(u^{\mathrm{d}}\right)$, we consider the following Stokes problem:

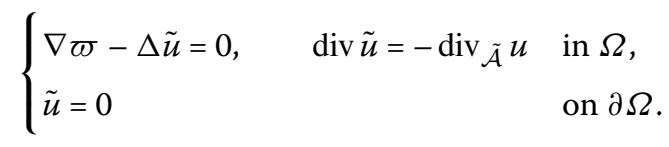

Using the existence theory of Stokes problem, we get a solution $(\tilde{u}, \varpi) \in H_{0}^{3} \times H^{2}$. Moreover,

$$
\|\tilde{u}\|_{3} \lesssim\left\|\operatorname{div}_{\tilde{\mathcal{A}}} u\right\|_{2} \lesssim \delta^{2} e^{2 \Lambda t}
$$

We define that $v^{\mathrm{d}}:=u^{\mathrm{d}}-\tilde{u}$, then it is easy to see that $v^{\mathrm{d}} \in H_{\sigma}^{3}$. Applying (2.2) to $-E\left(v^{\mathrm{d}}\right)$, we get

$$
-E\left(v^{\mathrm{d}}\right) \leq \Lambda^{2}\left\|\sqrt{\bar{\rho}} v^{\mathrm{d}}\right\|_{0}^{2}+\Lambda \mu\left\|\nabla v^{\mathrm{d}}\right\|_{0}^{2}
$$

which, together with (5.1) and (5.14), immediately implies

$$
-E\left(u^{\mathrm{d}}\right) \leq \Lambda^{2}\left\|\sqrt{\bar{\rho}} u^{\mathrm{d}}\right\|_{0}^{2}+\Lambda \mu\left\|\nabla u^{\mathrm{d}}\right\|_{0}^{2}+c \delta^{3} e^{3 \Lambda t} .
$$

Inserting it into (5.13), we arrive at

$$
\left\|\sqrt{\bar{\rho}} u_{t}^{\mathrm{d}}\right\|_{0}^{2}+2 \mu \int_{0}^{t}\left\|\nabla_{\mathcal{A}} u_{\tau}^{\mathrm{d}}\right\|_{0}^{2} \mathrm{~d} \tau \leq \Lambda^{2}\left\|\sqrt{\bar{\rho}} u^{\mathrm{d}}\right\|_{0}^{2}+\Lambda \mu\left\|\nabla u^{\mathrm{d}}\right\|_{0}^{2}+c \delta^{3} e^{3 \Lambda t}
$$

In addition,

$$
\mu \int_{0}^{t}\left\|\nabla u_{\tau}^{\mathrm{d}}\right\|_{0}^{2} \mathrm{~d} \tau \leq \mu \int_{0}^{t}\left(\left\|\nabla_{\mathcal{A}} u_{\tau}^{\mathrm{d}}\right\|_{0}^{2}+\|\tilde{\mathcal{A}}\|_{2}\left\|u_{\tau}^{\mathrm{d}}\right\|_{1}^{2}\right) \mathrm{d} \tau \leq \mu \int_{0}^{t}\left\|\nabla_{\mathcal{A}} u_{\tau}^{\mathrm{d}}\right\|_{0}^{2} \mathrm{~d} \tau+c \delta^{3} e^{3 \Lambda t}
$$

Furthermore, we can deduce from (5.15) that

$$
\left\|\sqrt{\bar{\rho}} u_{t}^{\mathrm{d}}\right\|_{0}^{2}+2 \mu \int_{0}^{t}\left\|\nabla u_{\tau}^{\mathrm{d}}\right\|_{0}^{2} \mathrm{~d} \tau \leq \Lambda^{2}\left\|\sqrt{\bar{\rho}} u^{\mathrm{d}}\right\|_{0}^{2}+\Lambda \mu\left\|\nabla u^{\mathrm{d}}\right\|_{0}^{2}+c \delta^{3} e^{3 \Lambda t} .
$$

Recalling the initial data $u^{\mathrm{d}}(0)=\delta^{2} u^{\mathrm{r}}$, by exploiting Newton-Leibniz's formula and Young's inequality, we find that

$$
\begin{aligned}
\Lambda \mu\left\|\nabla u^{\mathrm{d}}\right\|_{0}^{2} & =2 \Lambda \int_{0}^{t} \int \mu \nabla u^{\mathrm{d}}: \nabla u_{\tau}^{\mathrm{d}} \mathrm{d} y \mathrm{~d} \tau+\delta^{4} \Lambda \mu\left\|\nabla u^{\mathrm{r}}\right\|_{0}^{2} \\
& \leq \Lambda^{2} \mu \int_{0}^{t}\left\|\nabla u^{\mathrm{d}}\right\|_{0}^{2} \mathrm{~d} \tau+\mu \int_{0}^{t}\left\|\nabla u_{\tau}^{\mathrm{d}}\right\|_{0}^{2} \mathrm{~d} \tau+c \delta^{3} e^{3 \Lambda t} .
\end{aligned}
$$


Then we derive from (5.16) that

$$
\frac{1}{\Lambda}\left\|\sqrt{\bar{\rho}} u_{t}^{\mathrm{d}}\right\|_{0}^{2}+\mu\left\|\nabla u^{\mathrm{d}}\right\|_{0}^{2} \leq \Lambda\left\|\sqrt{\bar{\rho}} u^{\mathrm{d}}\right\|_{0}^{2}+2 \Lambda \mu \int_{0}^{t}\left\|\nabla u^{\mathrm{d}}\right\|_{0}^{2} \mathrm{~d} \tau+c \delta^{3} e^{3 \Lambda t} .
$$

In addition,

$$
\frac{\mathrm{d}}{\mathrm{d} t}\left\|\sqrt{\bar{\rho}} u^{\mathrm{d}}\right\|_{0}^{2}=2 \int \bar{\rho} u^{\mathrm{d}} \cdot u_{t}^{\mathrm{d}} \mathrm{d} y \leq \frac{1}{\Lambda}\left\|\sqrt{\bar{\rho}} u_{t}^{\mathrm{d}}\right\|_{0}^{2}+\Lambda\left\|\sqrt{\bar{\rho}} u^{\mathrm{d}}\right\|_{0}^{2} .
$$

Putting the previous two estimates together, we get the differential inequality

$$
\frac{\mathrm{d}}{\mathrm{d} t}\left\|\sqrt{\bar{\rho}} u^{\mathrm{d}}\right\|_{0}^{2}+\mu\left\|\nabla u^{\mathrm{d}}\right\|_{0}^{2} \leq 2 \Lambda\left(\left\|\sqrt{\bar{\rho}} u^{\mathrm{d}}\right\|_{0}^{2}+\mu \int_{0}^{t}\left\|\nabla u^{\mathrm{d}}\right\|_{0}^{2} \mathrm{~d} \tau\right)+c \delta^{3} e^{3 \Lambda t} .
$$

Recalling $u^{\mathrm{d}}(0)=\delta^{2} u^{\mathrm{r}}$, we apply Gronwall's inequality to (5.18) to deduce that

$$
\left\|\sqrt{\bar{\rho}} u^{\mathrm{d}}\right\|_{0}^{2}+\mu \int_{0}^{t}\left\|\nabla u^{\mathrm{d}}\right\|_{0}^{2} \mathrm{~d} \tau \lesssim e^{2 \Lambda t}\left(\int_{0}^{t} \delta^{3} e^{3 \Lambda t} e^{-2 \Lambda \tau} \mathrm{d} \tau+\delta^{4}\left\|u^{\mathrm{r}}\right\|_{0}^{2}\right) \lesssim \delta^{3} e^{3 \Lambda t} .
$$

Further, we can infer from (5.16), (5.17), (5.19), and Friedrichs's inequality that

$$
\left\|u^{\mathrm{d}}\right\|_{1}^{2}+\left\|u_{t}^{\mathrm{d}}\right\|_{0}^{2}+\left\|u_{\tau}^{\mathrm{d}}\right\|_{L^{2}\left((0, t), H^{1}\right)}^{2} \lesssim \delta^{3} e^{3 \Lambda t} .
$$

Now we turn to deriving the error estimate for $\eta^{\mathrm{d}}$. It follows from (5.4) ${ }_{1}$ that

$$
\frac{\mathrm{d}}{\mathrm{d} t}\left\|\eta^{\mathrm{d}}\right\|_{1}^{2} \lesssim\left\|u^{\mathrm{d}}\right\|_{1}\left\|\eta^{\mathrm{d}}\right\|_{1}
$$

Thus, using (5.20) and the initial data $\eta^{\mathrm{d}}(0)=\delta^{2} \eta^{\mathrm{r}}$, it follows that

$$
\left\|\eta^{\mathrm{d}}\right\|_{1} \lesssim \int_{0}^{t}\left\|u^{\mathrm{d}}\right\|_{1} \mathrm{~d} \tau+\delta^{2}\left\|\eta^{\mathrm{r}}\right\|_{1} \lesssim \sqrt{\delta^{3} e^{3 \Lambda t}}
$$

Noting that $L^{2} \hookrightarrow L^{1}$ and $H^{1} \hookrightarrow W^{1,1}$, then we can derive (5.3) from (5.20) and (5.21). This completes the proof of Proposition 5.1.

\section{Existence of escape times}

Let $\delta<\delta_{0},\left(\eta^{\mathrm{d}}, u^{\mathrm{d}}\right)$ be defined in Sect. 5 and $(\eta, u)$ be the classical solution constructed in Sect. 5 with an existence time $\left[0, T^{\max }\right)$. Let $\epsilon_{0} \in(0,1)$ be a constant, which will be defined in (6.7). We define

$$
\begin{aligned}
& T^{\delta}:=\Lambda^{-1} \ln \left(\epsilon_{0} / \delta\right)>0, \quad \text { i.e., } \quad \delta e^{\Lambda T^{\delta}}=\epsilon_{0}, \\
& T^{*}:=\sup \left\{t \in\left(0, T^{\max }\right) \mid \sqrt{\|\eta(\tau)\|_{4}^{2}+\|u(\tau)\|_{3}^{2}} \leq 2 C_{3} \delta_{0} \text { for any } \tau \in[0, t)\right\}, \\
& T^{* *}:=\sup \left\{t \in\left(0, T^{\max }\right) \mid\|(\eta, u)(\tau)\|_{0} \leq 2 C_{3} \delta e^{\Lambda \tau} \text { for any } \tau \in[0, t)\right\} .
\end{aligned}
$$

Noting that

$$
\left.\sqrt{\|\eta(t)\|_{4}^{2}+\|u(t)\|_{3}^{2}}\right|_{t=0}=\sqrt{\left\|\eta_{0}^{\delta}\right\|_{4}^{2}+\left\|u_{0}^{\delta}\right\|_{3}^{2}} \leq C_{3} \delta<2 C_{3} \delta_{0} \leq \delta_{2} \text { or } \delta_{3}
$$


thus $T^{*}>0, T^{* *}>0$ by Proposition 3.1. Moreover, by Proposition 3.1 and Remark 3.2, it is easy to see that

$$
\begin{aligned}
& \sqrt{\left\|\eta\left(T^{*}\right)\right\|_{4}^{2}+\left\|u\left(T^{*}\right)\right\|_{3}^{2}}=2 C_{3} \delta_{0}, \quad \text { if } T^{*}<\infty, \\
& \left\|(\eta, u)\left(T^{* *}\right)\right\|_{0}=2 C_{3} \delta e^{\Lambda T^{* *}}, \quad \text { if } T^{* *}<T^{\max } .
\end{aligned}
$$

We denote $T^{\min }:=\min \left\{T^{\delta}, T^{*}, T^{* *}\right\}$. Noting that $(\eta, u)$ satisfies

$$
\sup _{0 \leq t<T_{\min }} \sqrt{\|\eta(t)\|_{4}^{2}+\|u(t)\|_{3}^{2}} \leq \delta_{1}
$$

thus, for any $t \in\left(0, T^{\min }\right),(\eta, u)$ enjoys (3.51) and (3.52) with $\left(\eta_{0}^{\delta}, u_{0}^{\delta}\right)$ in place of $\left(\eta^{0}, u^{0}\right)$ by the second conclusion in Proposition 3.1. Since $\|(\eta, u)(t)\|_{0} \leq 2 C_{3} \delta e^{\Lambda t}$ on $\left(0, T^{\mathrm{min}}\right)$, from estimate (3.51) and (6.2), we derive that, for all $t \in\left(0, T^{\mathrm{min}}\right)$,

$$
\mathfrak{E}(t)+\int_{0}^{t} \mathcal{D}(\tau) \mathrm{d} \tau \leq c_{0} \delta^{2} e^{2 \Lambda t}+\Lambda \int_{0}^{t} \mathfrak{E}(\tau) \mathrm{d} \tau
$$

for some positive constant $c_{0}$. Applying Gronwall's inequality to (6.5), we arrive at

$$
\mathfrak{E}(t) \lesssim \delta^{2}\left(e^{2 \Lambda t}+\Lambda \int_{0}^{t} e^{\Lambda(t+\tau)} \mathrm{d} \tau\right) \lesssim \delta^{2} e^{2 \Lambda t}
$$

Inserting the above estimate to (6.5), we have

$$
\mathfrak{E}(t)+\int_{0}^{t} \mathcal{D}(\tau) \mathrm{d} \tau \lesssim \delta^{2} e^{2 \Lambda t}
$$

which, together with (3.52), yields that, for some constant $C_{5}$,

$$
\mathcal{E}(t)+\int_{0}^{t} \mathcal{D}(\tau) \mathrm{d} \tau \leq\left(C_{5} \delta e^{\Lambda t}\right)^{2} \leq C_{5}^{2} \epsilon_{0}^{2} .
$$

We define

$$
m_{0}:=\min _{\tilde{\chi}^{0}=\tilde{\eta}^{0}, \tilde{u}^{0}}\left\{\left\|\tilde{\chi}_{\mathrm{h}}^{0}\right\|_{L^{1}},\left\|\tilde{\chi}_{3}^{0}\right\|_{L^{1}}\right\}
$$

and

$$
\epsilon_{0}:=\min \left\{\frac{\theta}{C_{5}}, \frac{C_{3} \delta_{0}}{C_{5}}, \frac{C_{3}^{2}}{4 C_{4}^{2}}, \frac{m_{0}^{2}}{4 C_{4}^{2}}\right\}>0,
$$

where $\theta$ and $C_{4}$ come from Proposition 5.1 with $\gamma=C_{5}$ and $\beta=\epsilon_{0}$. By (4.3), $m_{0}>0$. Noting that $(\eta, u)$ satisfies (6.6), $\epsilon_{0} \leq \theta / C_{5}$ and $\beta=\epsilon_{0}$, then, by Proposition 5.1 with $\gamma=C_{5}$, we directly have (5.3) for any $t \in\left(0, T^{\mathrm{min}}\right)$. Furthermore, we get the relation

$$
T^{\delta}=T^{\min } \neq T^{*} \text { or } T^{* *}
$$

which can be proved by contradiction as follows: 
If $T^{\mathrm{min}}=T^{*}$, then $T^{*}<\infty$. Noting that $\epsilon_{0} \leq C_{3} \delta_{0} / C_{5}$, thus we infer from (6.6) that

$$
\sqrt{\left\|\eta\left(T^{*}\right)\right\|_{4}^{2}+\left\|u\left(T^{*}\right)\right\|_{3}^{2}} \leq C_{3} \delta_{0}<2 C_{3} \delta_{0}
$$

which contradicts (6.3). So, $T^{\min } \neq T^{*}$. Likewise, if $T^{\min }=T^{* *}$, then $T^{* *}<T^{*} \leq T^{\max }$. Since $\sqrt{\epsilon_{0}} \leq C_{3} / 2 C_{4}$, then we can infer from (4.1), (5.3), (6.1), and the fact $\epsilon_{0} \leq C_{3}^{2} / 4 C_{4}^{2}$ that

$$
\begin{aligned}
\left\|(\eta, u)\left(T^{* *}\right)\right\|_{0} & \leq\left\|\left(\eta^{\mathrm{a}}, u^{\mathrm{a}}\right)\left(T^{* *}\right)\right\|_{0}+\left\|\left(\eta^{\mathrm{d}}, u^{\mathrm{d}}\right)\left(T^{* *}\right)\right\|_{0} \leq \delta e^{\Lambda T^{* *}}\left(C_{3}+C_{4} \sqrt{\delta e^{\Lambda T^{* *}}}\right) \\
& \leq \delta e^{\Lambda T^{* *}}\left(C_{3}+C_{4} \sqrt{\epsilon_{0}} \leq 3 C_{3} \delta e^{\Lambda T^{* *}} / 2<2 C_{3} \delta e^{\Lambda T^{* *}}\right.
\end{aligned}
$$

which contradicts (6.4). Thus, $T^{\mathrm{min}} \neq T^{* *}$. This completes the proof of (6.8).

By (6.8) and the definition of $T^{\min }$, we have that $T^{\delta}<T^{*} \leq T^{\max }$. By (6.6) and the fact $\epsilon_{0} \leq \delta_{2} / C_{5}$, we have that $\sqrt{\|\eta(t)\|_{4}^{2}+\|u(t)\|_{3}^{2}} \leq \delta_{2}$. Then we can see that $(\eta, u) \in$ $C^{0}\left([0, T], H_{0}^{3} \times H_{0}^{2}\right)$ for some $T \in\left(T^{\delta}, T^{\max }\right)$, and $(\eta, u, q)$ also enjoys the regularity mentioned in Proposition 3.1.

Noting that $\sqrt{\epsilon_{0}} \leq m_{0} / 2 C_{4}$ and (5.3) holds for $t=T^{\delta}$, then making use of (4.1), (5.3), and (6.1), we immediately deduce that

$$
\begin{aligned}
\left\|\chi\left(T^{\delta}\right)\right\|_{L^{1}} & \geq\left\|\chi^{\mathrm{a}}\left(T^{\delta}\right)\right\|_{L^{1}}-\left\|\chi^{\mathrm{d}}\left(T^{\delta}\right)\right\|_{L^{1}} \\
& >\delta e^{\Lambda T^{\delta}}\left(\left\|\tilde{\chi}^{0}\right\|_{L^{1}}-C_{4} \sqrt{\delta e^{\Lambda T^{\delta}}}\right) \geq\left(m_{0}-C_{4} \sqrt{\epsilon_{0}}\right) \epsilon_{0} \geq m_{0} \epsilon_{0} / 2
\end{aligned}
$$

where $\chi=\eta_{3}, \eta_{\mathrm{h}}, u_{3}$ or $u_{\mathrm{h}}$. This completes the proof of Theorem 1.1 by taking $\epsilon:=m_{0} \epsilon_{0} / 2$.

\section{Conclusion}

In this paper, we prove the existence of classical solutions of RT instability in $L^{1}$-norm in Lagrangian coordinates based on a bootstrap instability method with finer analysis, if $\kappa$ is less than the threshold $\kappa_{\mathrm{C}}$. Moreover, we also get a unique classical solution for the RT instability in $L^{1}$-norm in Eulerian coordinates by further applying an inverse transformation of Lagrangian coordinates. Our instability result in Eulerian coordinates improves the known one that the solutions of RT instability shall be in $H^{2}$-norm in [25].

\section{Acknowledgements}

The authors would like to thank the anonymous referee for invaluable suggestions, which improved the presentation of this paper.

Funding

No funding.

Abbreviations

Not applicable.

Availability of data and materials

Not applicable.

Competing interests

The authors declare that they have no competing interests.

\section{Authors' contributions}

This work was carried out in collaboration between both authors. WW designed the study and guided the research. ZT performed the analysis and wrote the first draft of the manuscript. WW and ZT managed the analysis of the study. Both authors read and approved the final manuscript. 


\section{Publisher's Note}

Springer Nature remains neutral with regard to jurisdictional claims in published maps and institutional affiliations.

\section{Received: 8 May 2019 Accepted: 9 September 2019 Published online: 18 September 2019}

\section{References}

1. Adams, R.A., John, J.F.F.: Sobolev Space. Academic Press, New York (2005)

2. Bhatti, M.M., Abbas, M.A., Rashidi, M.M.: A robust numerical method for solving stagnation point flow over a permeable shrinking sheet under the influence of MHD. Appl. Math. Comput. 316, 381-389 (2017)

3. Chandrasekhar, S.: Hydrodynamic and Hydromagnetic Stability. The International Series of Monographs on Physics. Clarendon, Oxford (1961)

4. Chen, Y.P., Wang, W.W., Zhao, Y.Y.: On effects of elasticity and magnetic fields in the linear Rayleigh-Taylor instability of stratified fluids. J. Inequal. Appl. 2018, 203 (2018)

5. Duan, R., Jiang, F., Jiang, S.: On the Rayleigh-Taylor instability for incompressible, inviscid magnetohydrodynamic flows. SIAM J. Appl. Math. 71, 1990-2013 (2011)

6. Duan, R., Jiang, F., Yin, J.P.: Rayleigh-Taylor instability for compressible rotating flows. Acta Math. Sci. Ser. B Engl. Ed. 35 , 1359-1385 (2015)

7. Friedlander, S., Strauss, W., Vishik, M.: Nonlinear instability in an ideal fluid. Ann. Inst. Henri Poincaré, Anal. Non Linéaire $14,187-209(1997)$

8. Groza, G., Ali Khan, S.M., Pop, N.: Approximate solutions of boundary value problems for ODEs using Newton interpolating series. Carpath. J. Math. 25, 73-81 (2009)

9. Guo, Y., Hallstrom, C., Spirn, D.: Dynamics near unstable, interfacial fluids. Commun. Math. Phys. 270, 635-689 (2007)

10. Guo, Y., Strauss, W.A.: Instability of periodic BGK equilibria. Commun. Pure Appl. Math. 48, 861-894 (1995)

11. Guo, Y., Strauss, W.A.: Nonlinear instability of double-humped equilibria. Ann. Inst. Henri Poincaré, Anal. Non Linéaire 12, 339-352 (1995)

12. Guo, Y., Tice, I.: Linear Rayleigh-Taylor instability for viscous, compressible fluids. SIAM J. Math. Anal. 42, 1688-1720 (2011)

13. Guo, Y., Tice, I.: Almost exponential decay of periodic viscous surface waves without surface tension. Arch. Ration. Mech. Anal. 207, 459-531 (2013)

14. Hassan, M., Marin, M., Ellahi, R., Alamri, S.Z.: Exploration of convective heat transfer and flow characteristics synthesis by Cu-Ag/water hybrid-nanofluids. Heat Transf. Res. 49, 1837-1848 (2018)

15. Huang, G.J., Jiang, F., Wang, W.W.: On the nonlinear Rayleigh-Taylor instability of nonhomogeneous incompressible viscoelastic fluids under $L^{2}$-norm. J. Math. Anal. Appl. 455, 873-904 (2017)

16. Hwang, H.J., Guo, Y.: On the dynamical Rayleigh-Taylor instability. Arch. Ration. Mech. Anal. 167, 235-253 (2003)

17. Jang, J., Tice, I., Wang, Y.J.: The compressible viscous surface-internal wave problem: stability and vanishing surface tension limit. Commun. Math. Phys. 343, 1039-1113 (2016)

18. Jiang, F., Jiang, S.: On instability and stability of three-dimensional gravity driven viscous flows in a bounded domain. Adv. Math. 264, 831-863 (2014)

19. Jiang, F., Jiang, S.: On linear instability and stability of the Rayleigh-Taylor problem in magnetohydrodynamics. J. Math. Fluid Mech. 17, 639-668 (2015)

20. Jiang, F., Jiang, S.: On the stabilizing effect of the magnetic fields in the magnetic Rayleigh-Taylor problem. SIAM J. Math. Anal. 50, 491-540 (2018)

21. Jiang, F., Jiang, S.: On magnetic inhibition theory in non-resistive magnetohydrodynamic fluids. Arch. Ration. Mech. Anal. 233, 749-798 (2019)

22. Jiang, F., Jiang, S.: Nonlinear stability and instability in the Rayleigh-Taylor problem of stratified compressible MHD fluids. Calc. Var. Partial Differ. Equ. 58(1), Art. 29 (2019)

23. Jiang, F., Jiang, S., Wang, W.W.: Nonlinear Rayleigh-Taylor instability for nonhomogeneous incompressible viscous magnetohydrodynamic fluids. Discrete Contin. Dyn. Syst., Ser. S 9, 1853-1898 (2016)

24. Jiang, F., Jiang, S., Wang, Y.J.: On the Rayleigh-Taylor instability for the incompressible viscous magnetohydrodynamic equations. Commun. Partial Differ. Equ. 39, 399-438 (2014)

25. Jiang, F., Jiang, S., Wu, G.C.: On stabilizing effect of elasticity in the Rayleigh-Taylor problem of stratified viscoelastic fluids. J. Funct. Anal. 272, 3763-3824 (2017)

26. Jiang, F., Jiang, S., Zhan, W.C.: Instability of the abstract Rayleigh-Taylor problem and applications (2018). arXiv:1811.11610

27. Jiang, F., Wu, G.C., Zhong, X.: On exponential stability of gravity driven viscoelastic flows. J. Differ. Equ. 260, 7498-7534 (2016)

28. Jiang, F., Zhao, Y.Y.: On classical solutions of Rayleigh-Taylor instability for inhomogeneous incompressible viscous fluids in bounded domains (2019). arXiv:1901.04999

29. Jiang, Y., Li, X.J., Zhao, Y.Y.: On the stability of Rayleigh-Taylor problem for stratified rotating viscoelastic fluids. Bound. Value Probl. 2018, Paper No. 122 (2018)

30. Liu, X.L., Song, F.Y., Xu, C.J.: An efficient spectral method for the inextensible immersed interface in incompressible flows. Commun. Comput. Phys. 25, 1071-1096 (2019)

31. Majda, A.J., Bertozzi, A.L.: Vorticity and Incompressible Flow. Cambridge University Press, Cambridge (2002)

32. Novotny, A., Straškraba, I.: Introduction to the Mathematical Theory of Compressible Flow. Oxford University Press, Oxford (2004)

33. Othman, M.I., Marin, M.: Effect of thermal loading due to laser pulse on thermoelastic porous medium under G-N theory. Results Phys. 7, 3863-3872 (2017)

34. Shao, Z.Q.: Riemann problem with delta initial data for the isentropic relativistic Chaplygin Euler equations. Z. Angew. Math. Phys. 67(3), Art. 66 (2016)

35. Shao, Z.Q:: The Riemann problem for the relativistic full Euler system with generalized Chaplygin proper energy density-pressure relation. Z. Angew. Math. Phys. 69(2), Art. 44 (2018)

36. Su, Y.H., Chen, L.Z., Li, X.J., Xu, C.J.: On the inf-sup constant of a triangular spectral method for the Stokes equations. Comput. Methods Appl. Math. 16, 507-522 (2016) 
37. Taylor, G.I.: The stability of liquid surface when accelerated in a direction perpendicular to their planes. Proc. R. Soc. A 201, 192-196 (1950)

38. Wang, W.W., Zhao, Y.Y.: On the Rayleigh-Taylor instability in compressible viscoelastic fluids. J. Math. Anal. Appl. 463, 198-221 (2018)

39. Wang, Y.J.: Critical magnetic number in the MHD Rayleigh-Taylor instability. J. Math. Phys. 53, 073701 (2012)

40. Wang, Y.J.: Sharp nonlinear stability criterion of viscous non-resistive MHD internal waves in 3D. Arch. Ration. Mech Anal. 231, 1675-1743 (2019)

41. Wang, Y.J., Tice, I., Kim, C.: The viscous surface-internal wave problem: global well-posedness and decay. Arch. Ration. Mech. Anal. 212, 1-92 (2014)

42. Zhao, Y.Y., Wang, W.W.: Nonlinear convective instability in the compressible magnetic convection problem without heat conductivity. J. Math. Anal. Appl. 467, 480-500 (2018)

Submit your manuscript to a SpringerOpen ${ }^{\circ}$ journal and benefit from:

- Convenient online submission

- Rigorous peer review

- Open access: articles freely available online

- High visibility within the field

- Retaining the copyright to your article

Submit your next manuscript at $\boldsymbol{\Delta}$ springeropen.com 